\title{
Tracking the Reorganization of Module Structure in Time-Varying Weighted Brain Functional Connectivity Networks
}

\author{
Christoph Schmidt*, Diana Piper and Britta Pester \\ Bernstein Group for Computational Neuroscience Jena \\ Institute of Medical Statistics, Computer Sciences and Documentation \\ Jena University Hospital, Friedrich Schiller University Jena \\ Bachstrasse 18, 07743 Jena, Germany \\ *christoph.schmidt@med.uni-jena.de \\ Andreas Mierau \\ Institute of Movement and Neurosciences \\ German Sport University Cologne, Am Sportpark Muengersdorf 6 \\ 50933 Cologne, Germany \\ Herbert Witte \\ Bernstein Group for Computational Neuroscience Jena \\ Institute of Medical Statistics, Computer Sciences and Documentation \\ Jena University Hospital, Friedrich Schiller University Jena \\ Bachstrasse 18, 07743 Jena, Germany
}

Accepted 14 October 2017

Published Online 29 December 2017

\begin{abstract}
Identification of module structure in brain functional networks is a promising way to obtain novel insights into neural information processing, as modules correspond to delineated brain regions in which interactions are strongly increased. Tracking of network modules in time-varying brain functional networks is not yet commonly considered in neuroscience despite its potential for gaining an understanding of the time evolution of functional interaction patterns and associated changing degrees of functional segregation and integration. We introduce a general computational framework for extracting consensus partitions from defined time windows in sequences of weighted directed edge-complete networks and show how the temporal reorganization of the module structure can be tracked and visualized. Part of the framework is a new approach for computing edge weight thresholds for individual networks based on multiobjective optimization of module structure quality criteria as well as an approach for matching modules across time steps. By testing our framework using synthetic network sequences and applying it to brain functional networks computed from electroencephalographic recordings of healthy subjects that were exposed to a major balance perturbation, we demonstrate the framework's potential for gaining meaningful insights into dynamic brain function in the form of evolving network modules. The precise chronology of the neural processing inferred with our framework and its interpretation helps to improve the currently incomplete understanding of the cortical contribution for the compensation of such balance perturbations.
\end{abstract}

Keywords: Time-varying network; weighted network analysis; thresholding procedures; module structure; network community; consensus clustering; module matching; brain connectivity.

\footnotetext{
This is an Open Access article published by World Scientific Publishing Company. It is distributed under the terms of the Creative Commons Attribution 4.0 (CC-BY) License. Further distribution of this work is permitted, provided the original work is properly cited.
} 


\section{Introduction}

Time-variant analysis of functional brain connectivity is a fast expanding field in computational neuroscience research that promises to elucidate large-scale brain network interactions ${ }^{1}$ Directed or undirected interactions in the sense of functional connectivity refer to statistical dependencies between the neuronal activities of brain regions, which cannot be measured directly.2.3 The computation of functional connectivity requires sophisticated analysis tools and modeling that use measured brain activity as input data $\stackrel{45}{4}$ Multivariate brain activity data with an appropriate time-resolution is a prerequisite for a time-variant interaction analysis ${ }^{4}$ Such data may be obtained, for example, from electroencephalography (EEG), magnetoencephalography (MEG), magnetic resonance encephalography recordings,$\frac{6}{6}$ and also, with limitations, from resting state functional magnetic resonance imaging (fMRI) scans $!^{7}$ The results of the time-variant connectivity analysis can be represented e.g. as a time-varying network given by a sequence of networks evolving during the investigated neural processing. Meaningful information about structural changes within such network sequences can be extracted by the application of network science methods 89

A feature of brain functional network topology that is of particular interest is its composition of distinct modules, which is often called community structure in other contexts ${ }^{[10]}$ From the prospect of our work, network modules are given by a natural partition of nodes into separate subsets, i.e. groups, so that member nodes of the same group have a high probability of being connected by an edge, whereas the probability of being connected is lower for nodes of different groups. Thus, nodes within a module maintain more and stronger interactions to each other than to nodes of different modules. Modules in brain functional networks can be interpreted in terms of functionally segregated and delineated brain areas, which yield important insights into the studied brain activity $\frac{13}{16}$ It can be assumed that the way interactions between functionally distinct brain regions are redistributed in the course of the neural information processing is reflected in the dynamic reconfiguration of module structure in time-varying brain functional networks $\frac{3113}{15}$ Still, in current neuroscience research, the analysis of the temporal reorganization of module structure is not commonly considered, despite its potential for improving the understanding of the time evolution of functional interaction patterns and the resulting functional segmentation of corresponding brain areas.

Our proposed computational framework encompasses all network data processing steps necessary to accomplish the task of tracking module structure reorganization. It has been primarily designed for temporal sequences of weighted directed edgecomplete networks, in which the continuous nature of interaction strengths and the direction of interactions are preserved. Thresholding is an essential operation for the analysis of brain functional networks, which contributes to the determination of their structural characteristics ${ }^{[17}$ For it, we present a novel method that computes meaningful networkspecific edge weight thresholds using multicriteria optimization of network topology characteristics. In this regard, it is one of the first published methods for identifying thresholds with respect to an optimization of the underlying structure of the network, $\frac{19}{19}$ which stands in contrast with constraining or fixing certain basic network properties $\frac{18}{18}$ We compare the thresholding approaches of our framework with other known threshold concepts. The framework incorporates two alternative approaches for processing the time-varying network data to extract a consensus partition from several defined time windows that cover regions of interest of the network sequence (Sec. 3.1). Thereby, different perspectives on the temporal evolution of module structure are offered by each of the two extraction approaches. The tracking of dynamic network module structure is based on the generated sequences of consensus partitions, which have to be further processed by a module matching algorithm (Sec. 3.4) to consistently color-code modules for the visualization of module structure changes by means of Sankey diagrams (alluvial diagrams) ${ }^{1420}$ and series of network plots (Sec. 3.5). We validated the computational framework (Sec. 4.1) using simulated network sequences with ground truth module structure (Sec. 2.1). Subsequently, we apply the framework to a real-world time-varying brain functional network obtained from EEG recordings of healthy subjects (Sec. 2.2). In the context of neuroscience, the computation of timevarying networks can be seen as a first computational step of the framework (Sec. 2.3). The underlying study investigates the currently only imprecisely 
understood contribution of the cerebral cortex to compensatory reactions following a sudden balance perturbation in order to regain postural stability. A previous study 21 that focused on time-variant connectivity analysis has explored the same data set and provides a priori information about suitable frequency bands and the dynamics of connectivity changes. The results demonstrate that in the transition from balancing on a stable surface (BSS) to an unstable surface (BUS), two topographically circumscribed, time-varying connectivity networks are established; one is associated with the theta and one with the alpha frequency band. Networks of the theta network sequence can be described as sets of delimited subnetworks comprising the frontal, central and parietal cortex with individual temporal and spatial developments within and between those substructures. The modular structure of the theta networks was the reason why we used only this time-varying network for module tracking. Within this scientific context, we demonstrate that the application of our framework yields novel insights into time-varying functional brain network organization and enables to identify interesting events in the cortical processing during the task execution (Sec. 4.2).

\section{Material}

\subsection{Synthetic time-varying network}

An overview of the simulation of a synthetic timevarying network $\mathcal{W}=\left(\mathcal{W}_{t}\right)_{t=1, \ldots, T}$ that consists of a sequence of $T$ network observations with ground truth module structure is given in Fig. 1. The connectivity structure of the $t$ th network observation is given by the edge weight matrix $\mathcal{W}_{t}$, where the strength of the directed interaction from node $i$ to

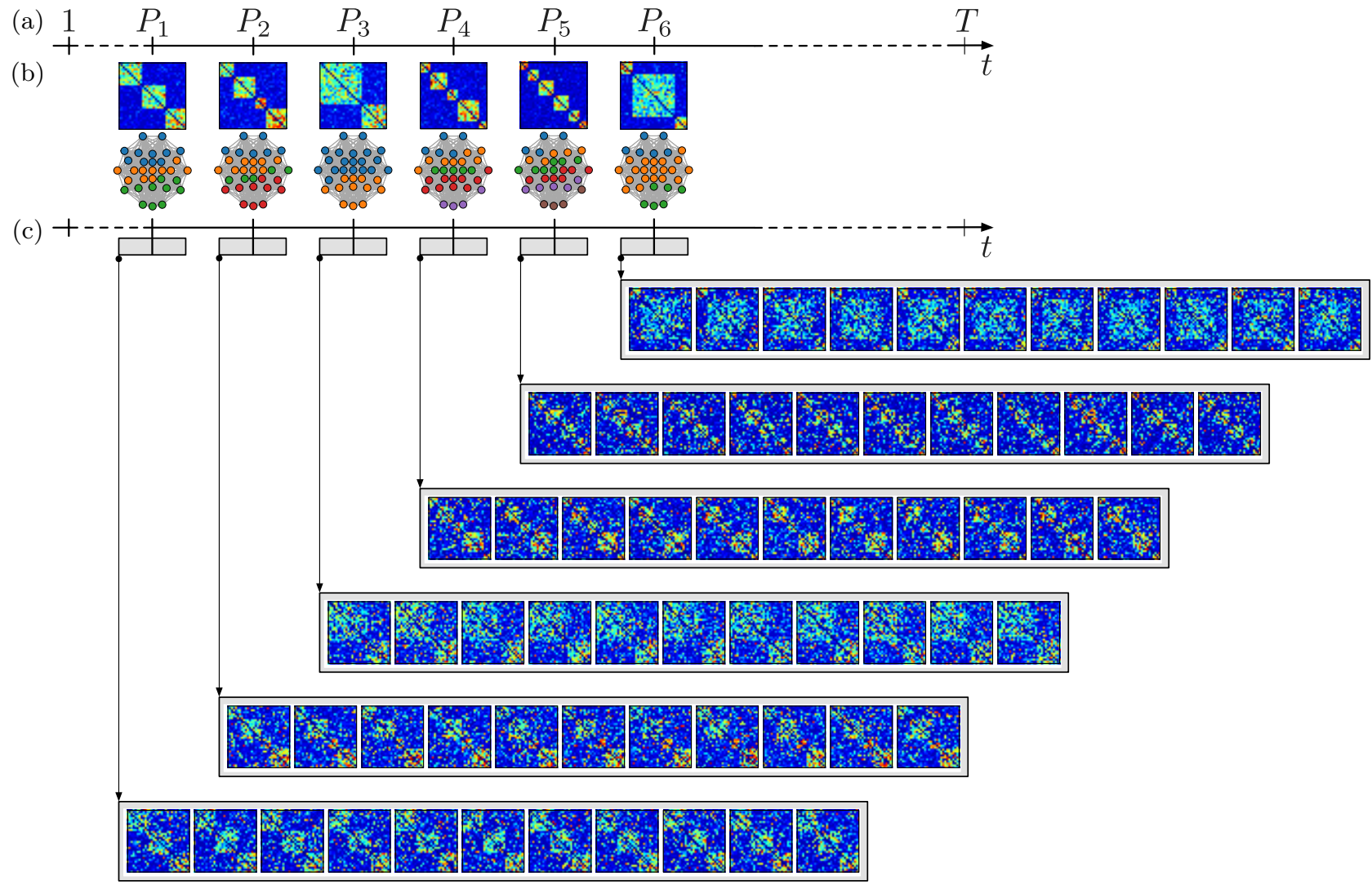

Fig. 1. Overview of the simulation of a synthetic network sequence with time-varying module structure. (a) The length of the network sequence is defined by the number of time grid points $P_{g}$, e.g. $g=1, \ldots, 6$, and the width of the time windows (e.g. 11 network observations) that are centered on these points. (b) For each point on the time grid, the edge weight matrix of an initial seed network with ground truth module structure is simulated. (c) The networks of individual time windows are generated by random perturbations of the edge weights of intra-module and inter-module interactions of the associated initial network. All networks generated during the simulations are weighted directed and edge-complete. 
node $j$ at time $t$ is indicated by $w_{i j}(t)$. The structure of the simulated time-varying network reflects how dynamic module structure reorganization is extracted by the application of the framework (see also Sec. 3.1). The network sequence of the timevarying network was simulated in a way that makes it possible to divide it into disjunct consecutive time windows (whose width might be given by an odd number of networks, for example 11 networks; Fig. 1(c)), which are centered on individual points $P_{g}$ of a defined time grid (we chose $g=1, \ldots, 6$; Fig. 1(a)). Thereby, the idea is that connectivity structure of networks within individual time windows should be aggregated to eventually obtain a sequence of consensus partitions that summarize stable features of the module structure encountered within each window. Thus, the simulation procedure starts with the simulation of an initial weighted directed edge-complete network with ground truth module structure (Fig. 1(b)) for each point on the time grid (Fig. 1(a)). Each of the initial networks has 32 nodes and contains two to six modules. They serve as seeds for the generation of the networks of the associated time window. The networks of the network sequence covered by each window (Fig. 1(c)) are simulated by weakening the definiteness of the associated initial network's ground truth module structure by means of introducing predefined levels of "interaction noise". This randomization is performed by selectively decreasing the weights $w_{i j}$ of a percentage of intra-module edges and independently increasing the weights of a (possibly different) percentage of inter-module edges of the initial network. All edge selections are performed uniformly at random. The simulated time-varying network shows major changes of its module structure composition at the transition from one window to another, whereas the dynamics of node module affiliations within windows is limited. The simulation algorithm outlined here is described in detail in Appendix B, where the resulting edge weight matrices are also shown.

\subsection{EEG acquisition}

The following description of the EEG acquisition is taken from our previous study 21 EEG data from 37 healthy male university students were analyzed. Participants were informed about the experimental protocol and their written consent was obtained beforehand. The study was designed and performed according to the standards set by the latest revision of the Declaration of Helsinki for medical research involving human subjects, and all procedures were approved by the Ethics Committee of the German Sport University. About 32 electrode positions, overlying the hole scalp and equally distributed over both hemispheres according to the 10-10 international system, were used to record the EEG data with a sampling rate of $1000 \mathrm{~Hz}$. The electrical reference was located at $\mathrm{FCz}$ and the ground electrode at AFz. Ocular artifacts were corrected using the ocular correction algorithm implemented in the "Brain Vision Analyzer 2" software. The data were rereferenced to the common average reference and downsampled to $100 \mathrm{~Hz}$. The downsampling was performed using the MATLAB decimate function, which implements a lowpass Chebyshev Type I IIR filter of order 8 . The influence of the filtering on timevariant functional connectivity measures was investigated by Leistritz et al. 23 where it was found that negative effects are unlikely for the frequency range investigated in this study.

The experiment ${ }^{24}$ consisted in 10 trials of transient unpredictable balance perturbations using a passively oscillating platform. Each perturbation trial, lasting $30 \mathrm{~s}$ in total, started immediately after the subjects positioned themselves on their dominant leg in the center of the platform and confirmed that they were "ready". The subjects were instructed to keep platform oscillations to a minimum after perturbation, while having their hands at the iliac crest and focusing on a fixation cross at eye level. The platform was laterally deflected by $2.2 \mathrm{~cm}$ and fixed by an anchor in this position. In each trial, the investigator randomly released the anchor within the 5 th and 20 th s, which induced a medial movement of the supportive platform. The inter-trial interval was $1 \mathrm{~min}$ in order to avoid fatigue. The perturbation onset was determined as the time point following anchor release at which the platform motion exceeded its mean oscillation level in medial-lateral or anteriorposterior direction during the last $5 \mathrm{~s}$ prior to anchor release by five standard deviations. All measurement systems were synchronized by an electrical pulse produced during anchor release. The EEG data were segmented into epochs of $8 \mathrm{~s}$ based on the switch from BSS to BUS, with $3 \mathrm{~s}$ before platform release and $5 \mathrm{~s}$ after. 


\subsection{Computation of time-varying brain functional networks}

We used scalp EEG sensor space data for the computation of time-varying edge-complete brain functional networks, where nodes correspond to the recording sites and are connected by weighted directed edges, which represent functional interactions between EEG activities recorded at the associated electrodes at the considered points in time. Time-variant amplitude spectra analysis Appendix D and directed connectivity analysis were carried out by means of a time-variant multivariate autoregressive (tvMVAR) model, which was estimated with the state-of-the-art Kalman filter approach of Milde et al. ${ }^{25}$ In addition to its advantage of time variance, this algorithm separately integrates multiple trials and thus, a loss of information due to averaging by trials prior to or after model estimation is avoided. Consequently, for multitrial data, it has been demonstrated that this algorithm has an overall superior performance in comparison with other Kalman approaches. ${ }^{26}$ For the tvMVAR modeling, the full electrode set consisting of 32 electrodes was used 21 The connectivity analysis based on the tvMVAR parameters was performed by means of the direct directed transfer function (dDTF) 27 The dDTF was chosen because of its good frequency resolution that enables to clearly separate specific frequency bands of interest. Van de Steen et al. ${ }^{[28}$ demonstrated that influences of volume conduction on DTF exist, i.e. the interpretation of our results must take this fact into consideration. Kaminski and Blinowska ${ }^{29}$ argued that in practice, this influence does not substantially distort the estimates.

The results of the $\mathrm{dDTF}$ can be represented by $D \cdot(D-1)$ time-frequency maps (dDTF-TFMs) for each interaction, where $D$ is the number of electrodes. Examples of dDTF-TFMs are shown in Fig. E.1 of the Appendix. We used results of a group analysis, i.e. grand mean dDTF-TFMs were obtained by averaging over all subjects. A theta frequency sub-band $(5-7 \mathrm{~Hz})$ was considered, as EEGactivity in this frequency band is associated with the balance task 2130 (see also Appendix E). The edge weight $w_{i j}(t)$ of the $t$ th network $\mathcal{W}_{t}$ of the resulting time-varying grand mean network $\mathcal{W}$ was computed by extracting the median value within the theta frequency sub-band at time $t$ from the corresponding grand mean dDTF-TFM. Subsequently, all edge weights were rescaled to improve the resolution of interaction strengths by enhancing strong interactions more than weak ones using

$$
f\left(w_{i j}(t), \mathcal{W}, b\right)=\left(\frac{w_{i j}(t)-\min (\mathcal{W})}{\max (\mathcal{W})-\min (\mathcal{W})}\right)^{b},
$$

where $b>0$ is the exponent of the mapping function ${ }^{31}$ We found heuristically that $b=1.5$ yields a good edge weight resolution for our data. According to the sampling frequency of $100 \mathrm{~Hz}$ and the duration of the analysis interval $(8 \mathrm{~s})$, the investigated timevarying network $\mathcal{W}$ consisted of 800 single networks that represent the connectivity structure at the corresponding points in time.

The influence of motion artifacts on the connectivity analysis was investigated in a previous study, 21 whose results can be summarized as follows. It must be considered that the interval ranging from $3 \mathrm{~s}$ to $3.5 \mathrm{~s}$ is strongly influenced by the BUS onset. For all other time ranges, the following conclusions can be made. For the considered theta sub-band, a contamination by higher harmonics of the "sway-related" motion interference (center-frequency $=2.6 \mathrm{~Hz}$ ) is unverifiable, so that a connectivity analysis for this frequency band is meaningful. This is true for all electrodes, except for $\mathrm{Fp} 1 / 2$ and $\mathrm{P} 7 / \mathrm{P} 8$. As a consequence of this analysis, electrodes Fp1, Fp2, P7 and P8, which acted solely as sources of interactions, were removed from the final time-varying network (see also Appendix D). Thus, 28 nodes were available for the module structure extraction and tracking. The layout of the EEG electrodes that were considered for the module tracking is shown in Appendix A. Fig. A.1

\section{Methods of the Computational Framework}

\subsection{Overview}

In the following, we give an overview of our proposed framework for extracting and tracking module structure reorganization in time-varying weighted directed edge-complete networks, i.e. in sequences of temporally related networks with the same fixed node set (Fig. 2). Individual networks in such a sequence correspond to distinct points in time $t$ and can be interpreted as observations of the interaction 


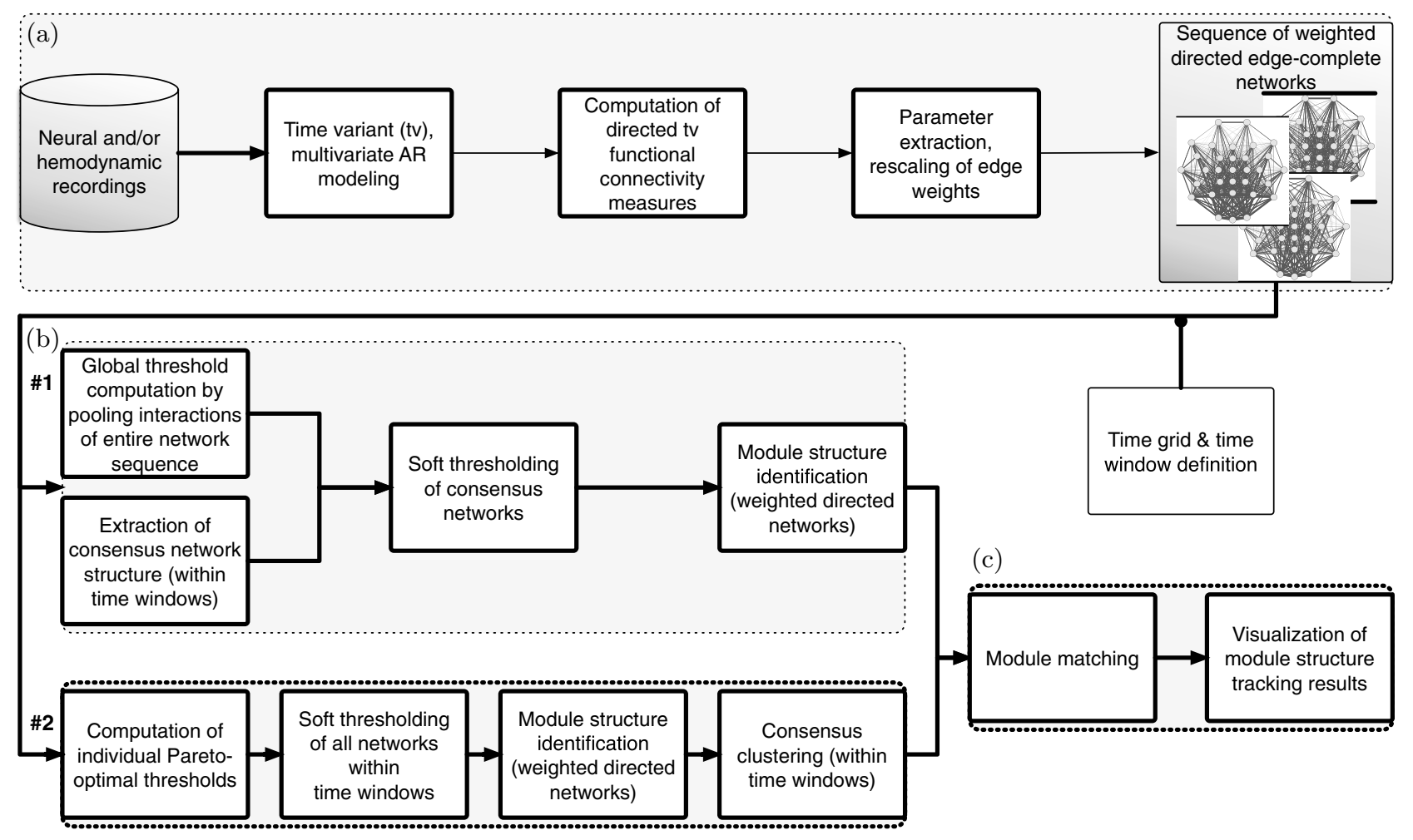

Fig. 2. Overview of the computational framework. (a) Computation of a time-varying network in the context of neuroscience from recordings of brain activity. (b) Two complementary approaches, \#1 and \#2, for extracting consensus partitions within time windows placed on the input network sequence. (c) Post-processing of the extracted consensus partitions to track and visualize module structure reorganization.

structure at the respective time. As already mentioned, the collection, computation and preprocessing of time-varying network data can be seen as preceding computational steps (Fig. 2(a)). In Sec. 2.3, we describe one way to compute a timevarying network in the context of neuroscience based on EEG data. We introduce two complementary module structure extraction approaches (\#1 and \#2, Fig. 2(b)) that use a time-varying network as input and offer different perspectives on the time evolution of network modules, which strongly depends on the applied thresholding procedure. The evolution of modules, i.e. their "life cycle", is defined in Appendix F. Meaningful structural changes in the network evolution have to be distinguished from fluctuations in the topology of single networks of the considered network sequence, which potentially cause a high temporal variation of identified module affiliations of nodes ${ }^{20132}$ For that purpose, specific intervals of the network sequence are extracted for the pooling of the interactions and module structures of the corresponding networks. This is performed by dividing the network sequence into nonoverlapping consecutive time windows, which are centered on individual points $P_{g}$ of a defined time grid, which determines regions of interest in the evolution of the studied process (cf. Fig. 1). Both module structure extraction approaches utilize specific strategies to soft threshold weighted networks (Fig. 2(b)). Soft thresholding refers to the removal of edges with weights lower than the threshold from the weighted network, whereas hard thresholding additionally dichotomizes the remaining edges to yield binary networks. The first time-varying module structure extraction approach (\#1) is based on computing consensus networks for all considered time windows, which are subsequently soft thresholded using multiple thresholds computed as percentiles of the edge weight distribution of the entire network sequence (see Sec. 3.2). After soft thresholding, a partition of nodes into modules is identified for each consensus network. The second approach of 
the framework (\#2) is based on a new method for computing network-specific thresholds (as opposed to systematically trying out multiple thresholds) by means of multiobjective optimization of module structure quality characteristics (see Sec. 3.3). Network partitions identified for all soft thresholded networks within each considered window are combined into a consensus partition using a consensus clustering approach ${ }^{33}$ Consequently, the difference between both analysis approaches is given particularly by the method used for the thresholding of edge weights and also by the way interaction information of the networks within time windows is aggregated to yield window-specific network partitions that summarize stable features of the module structure changes encountered within the windows. Because of the importance of the thresholding step for module tracking, the tracking results obtained by using our two new thresholding approaches were compared with other known concepts. Thereby, the global thresholding of approach \#1 is compared with the standard local multiple thresholding that accounts for the weights of only the network to be thresholded (\#1-local). The objective thresholding of approach \#2 is compared with another novel approach, efficiency cost optimization (ECO), that is like our approach \#2 based on computing a threshold that optimizes higher level structural properties of the resulting thresholded network, according to defined quality functions. ${ }^{[19}$ In their case, the quality function accounts for the trade-off between the efficiency ${ }^{34}$ of a network and its wiring cost. In the context of our framework, we applied ECO thresholding to the consensus network of each time window, similar to our approach \#1. The comparisons were carried out for both simulated and EEG data, where results related to \#1-local are only described and those which are related to ECO are described and illustrated (Figs. 5 and C.1).

Subsequently, the module matching algorithm that we introduce in Sec. 3.4 relates and tracks the time-varying modules to infer structural relationships between the partitions obtained at the considered time steps (Fig. 2(c)). Thereby, the module labels and corresponding color codes are adjusted. To gain an understanding of the investigated module structure reconfigurations, appropriate visualizations of the extracted and tracked dynamics have to be employed. For it, we use Sankey diagrams and EEG electrode layout network plots (Sec. 3.5).

\subsection{Module structure extraction approach \#1}

Consensus networks are constructed for all defined time windows. For each window, this construction is performed by computing the median weight of each individual edge in all covered networks, which conforms to a smoothing of the interactions found within that time window (cf. Figs. प(b) and प(c)). Edges with low weights, i.e. weak interactions, are removed from the still edge-complete consensus networks by means of soft thresholding them. For it, empirical multiple thresholding ${ }^{16 \text { 35:36 }}$ using a set of predefined edge weight percentiles as thresholds is combined with a pooling of the edge weights of all available networks of the entire network sequence. That is, instead of computing percentiles from the edge weight distribution of individual consensus networks (see our comparison approach), they are computed with respect to the distribution of edge weights observed at all available points in time. Thus, by using global edge weight percentiles as thresholds, the interaction structure of consensus networks at certain points on the time grid $P_{g}$ is contrasted with the global information on interaction strengths. After thresholding consensus networks usually have different numbers of weighted interactions. Using multiple thresholds necessitates the extraction of time evolving module structure separately for each obtained threshold. For the identification of module structure in the thresholded consensus networks, we used a generalization of the algorithm of Blondel et al. ${ }^{[37}$ for weighted directed networks. Isolated nodes that were disconnected from their network due to the thresholding are assigned the same module affiliation to exclude them from the interpretation of the results. This stands in contrast to the default output of most module detection algorithms that would assign these nodes to their own modules. In summary, application of approach \#1 reveals the reconfiguration of module structure with respect to deviations in the interaction strengths of single consensus networks from the global edge weight distribution, which is indicated by the extent of thresholding seen at different points on the time grid. 
As already mentioned in Sec. 3.1 the global threshold concept was compared with the standard local multiple thresholding using edge weight percentile values obtained from the weights of only the network to be thresholded.

\subsection{Module structure extraction approach \#2}

This approach utilizes a new concept for computing network-specific thresholds by means of a multiobjective optimization (Pareto optimization) of inherent module structure quality characteristics. The networks within given time windows are soft thresholded using thresholds computed as described below.

Given a weighted input network, a specified range of edge weight percentiles is considered and the corresponding edge weights are computed to serve as thresholds. For each threshold, a soft thresholding is performed and several module structure quality characteristics are computed for the resulting network if it remains at least weakly connected. In particular, the modularity measure, performance index (of the underlying binarized network) and coverage are computed $\frac{12 \mid 16}{16}$ Thereby, the network partition with maximum modularity $Q$ is identified by comparing the results of several module detection algorithms (walkTrap WU, ${ }^{38}$ infoMap WD, ${ }^{39}$ fast-

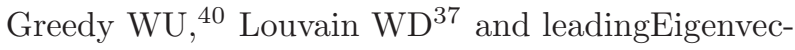
tor $\left.\mathrm{WD}^{411}\right)$. The evaluation of other characteristics is conceivable, e.g. the recently proposed module structure quality measure. ${ }^{42}$ The result of these computations is a vector for each threshold containing the corresponding values of all considered network characteristics. Thereby, the considered network characteristics represent independent and potentially conflicting objectives that have to be simultaneously optimized. Typically, there is no unique solution, i.e. a vector that is absolutely better in all objectives than any other. Thus, the goal of multiobjective optimization is to find solution vectors that represent a reasonable trade-off among the objectives $\stackrel{43 / 44}{ }$ All such vectors are known as Pareto-optimal solutions or nondominated points, because none of their objectives can be further improved without deteriorating the value of at least one other. The set of Paretooptimal solutions is called a Pareto front 4
A multiobjective optimization problem has the following form 43 :

$$
\begin{aligned}
& \operatorname{minimize}\left\{f_{1}(\boldsymbol{x}), f_{2}(\boldsymbol{x}), \ldots, f_{k}(\boldsymbol{x})\right\} \\
& \text { subject to } \boldsymbol{x} \in S
\end{aligned}
$$

The $k \geq 2$ objective functions (criteria) $f_{i}: S \rightarrow$ $\mathbb{R}$ quantify the values of the network characteristics of interest. The decision variable vector $\boldsymbol{x}=$ $\left(x_{1}, x_{2}, \ldots, x_{n}\right)^{T}$ is an element of the set $S$ of alternatives (feasible solutions) of the decision problem. $\stackrel{46}{6}$ For the problem of computing good edge weight thresholds, the decision variables are the single thresholds or, respectively, the corresponding soft thresholded networks. The dimension $k$ of the criterion space $\mathbb{R}^{k}$ is given by the number of objective functions $f=\left(f_{1}, \ldots, f_{k}\right)$. A decision variable vector $x^{*} \in S$ is efficient if there does not exist another decision variable vector $\boldsymbol{x} \in S$ such that $f_{i}(\boldsymbol{x}) \leq f_{i}\left(\boldsymbol{x}^{*}\right), \forall i=1, \ldots, k$ and $f_{j}(\boldsymbol{x})<f_{j}\left(\boldsymbol{x}^{*}\right)$ for at least one index $j$. An objective function vector (outcome) $\boldsymbol{f}^{*}=\left(f_{1}^{*}, \ldots, f_{k}^{*}\right)^{T}$ with $f_{i}^{*}=f_{i}\left(\boldsymbol{x}^{*}\right)$ is Pareto optimal (a nondominated point in the criterion space) if its corresponding decision variable vector is efficient.

The sign of the components of the resulting (objective function) vectors is interchanged to handle the situation that the underlying multicriteria optimization problem is formulated in terms of a maximization instead of a minimization problem. The Pareto front is computed based on pairwise comparisons and nondominated sorting.44/47 To obtain a single consensus solution, the centroid of the nondominated points of the Pareto front is computed. Finally, the nondominated point with minimum Euclidean distance to the centroid is considered as the best compromise and the corresponding threshold is returned as the final solution of the optimization problem.

Instead of computing a consensus network for each time window and identifying its module structure just like for approach \#1, a consensus partition ${ }^{33}$ for each time window is computed on the basis of the single partitions of all networks within a time window. Hence, it summarizes consistent features and trends in the network partitions in the vicinity of any considered point $P_{g}$ on the time grid. Again, a generalization of the algorithm of Blondel 
et al ${ }^{37}$ for weighted directed networks was used for all network module structure identifications during the consensus clustering. In summary, application of approach \#2 offers a strictly local view on the module structure evolution and reveals stable features of the module structure encountered in separate time windows.

As already mentioned in Sec. 3.1, the effects of network-specific, Pareto optimal thresholds on the time-varying module structure were compared with the ones of the ECO thresholding approach $\frac{19}{19}$ which is also based on a topological criterion.

\subsection{Optimal cost matching of modules}

Matching modules among network partitions at considered points in time are integral to inferring the time evolution of a dynamic network's module structure (see also Sec. 3.5). However, the problem of dealing with mismatched module labels is a general one that also appears in other contexts. Due to the randomized nature of network module detection algorithms, mismatched module labels are often encountered when analyzing different network partitions, which complicates analyses and graphical representation of module structure and its changes. In this section, we describe a global optimal cost matching of modules (module labels) in different network partitions to a reference partition, followed by a rematching of nonplausibly matched modules (module labels) among nonreference partitions.

To compute optimal cost module matchings across networks of a network sample, we propose the following algorithm that is based on the classical two-dimensional $(n: n)$-assignment problem with quadratic cost matrix ${ }^{\frac{48}{7}}$ and the unbalanced assignment problem with rectangular cost matrix, where two sets of entities with different cardinality have to be matched in a least-cost way $\sqrt[49]{51}$ As primary input parameter the module matching algorithm accepts a matrix of node module affiliations for at least two different network partitions with the same number of nodes. Formally, this kind of considered assignment problem can be stated as a linear program (see also Appendix G), which is solved with the mixed integer linear programming solver lpSolve.

The first computational step of the module matching algorithm is selecting the network partition with the maximum number of modules as a reference against which the module affiliations of all other networks are being matched separately. Then all pairs of modules of the reference partition and each nonreference partition are compared systematically to compute a cost matrix $C$ for every single reference partition-nonreference partition comparison. In detail, this cost matrix $C$ is defined as the difference between the number of network nodes and the cardinality of the intersection of member nodes of each considered module pair. Consequently, the costs for matching a reference network module to a pair of reference-nonreference network modules are decreasing with an increasingly larger overlap of module member nodes. Using this cost matrix, the assignment problem is solved. According to the solution obtained, module labels of each nonreference network partition are changed to the assigned module labels of the reference network partition, if the respective pairs of matched reference-nonreference modules have a minimum user-defined similarity, given by the matching threshold $\theta \in[0,1]$. The similarity of a pair of modules and their member nodes might be measured in terms of the relative overlap of module member nodes, i.e. by using the Jaccard coefficient of two sets $J\left(\mathcal{M}_{i}, \mathcal{M}_{k}\right)=\left|\mathcal{V}_{\mathcal{M}_{i}} \cap \mathcal{V}_{\mathcal{M}_{k}}\right| / \mid \mathcal{V}_{\mathcal{M}_{i}} \cup$ $\mathcal{V}_{\mathcal{M}_{k}} \mid$. Controlling for the similarity of each pair of reference-nonreference modules prevent low-quality assignments that could happen otherwise, despite the optimal cost nature of the computed assignments, as ultimately all nonreference modules are assigned to a reference partition module. Consequently, assignments of dissimilar modules are ignored. In this case, under the assumption of the novelty of the nonreference partition module and its composition of nodes, a new label (and thus a new color), which is not already in use, is allocated. The new color might be selected in a way that similar modules, e.g. two related modules that occurred as the result of a splitting event of a larger module, are assigned similar colors, i.e. by adjusting their hue or opacity. To account for the situation that modules of different nonreference partitions with similar member node composition got assigned different new unique labels as they could not be matched with sufficient quality to a module of the reference partition, a rematching is performed. For this rematching, pairs of previously nonmatched modules with assigned 
new labels are compared across all nonreference partitions. If the similarity of any compared module pair is above the matching threshold $\theta$, their module labels are adjusted to be the smallest of the two former labels. Thereby, it is important to keep track of all such rematched module pairs as subsequent rematchings with other modules that were not previously matched to the reference partition might further reduce the module labels, so that further label adjustments of already rematched modules become necessary. The rematching step improves the accuracy of the first global matching step and reduces the number of different newly assigned labels.

Since nodes in functional brain networks are associated with unique identity labels (e.g. EEG electrode identifiers or voxel positions), module structure of several networks should be primarily interpreted with respect to the spatial information of nodes and their location in the network, while the labels (colors) associated to the modules are acting merely as a guide.

\subsection{Visualization of the module structure evolution}

Sankey diagrams $\sqrt{14 \mid 20}$ represent a suitable means to visualize the temporal evolution of module structure. They display the time-varying module memberships of nodes in the form of different amounts of node flows between modules at different time steps. This graphical representation gives a good overview over long-term trends in the module affiliations of nodes and it shows time steps with large fluctuations and rapid reconfigurations of module structure. Thus, Sankey diagrams aid in discerning persistent, interesting modules from short-lived noisy ones and they allow for the identification of change points related to specific events, i.e. short periods in which the dynamics produces big changes in the network structure. In addition to the Sankey diagrams, network plots using the EEG electrode layout delineate the spatial location of modules and their affiliated nodes.

\section{Results}

\subsection{Simulated time-varying networks}

For testing purposes, we applied our two approaches for the extraction of time-varying module structure to a sequence of simulated networks with ground truth module structure. As described in Sec. 2.1, this network sequence consisted of different regions, i.e. time windows, in which the topology of individual networks reflects a common module structure given by an initial network (Figs. 1(b), 3(a) and B.1(a)), whose topology is strongly disturbed by "interaction noise" generated by a targeted randomization of intra- and inter-module edge weights (Figs. 1(c) and B.1(b)). We assessed the recoverability of module structure within the simulated time windows for various randomization levels. The extent to which the ground truth consensus partitions could be recovered from the time windows was quantified using the normalized variation of information (VI) as a partition distance measure ${ }^{[53}$ For the thresholding of module structure extraction approach \#1, the 80th percentiles of all available edge weights turned out to yield optimal results (see also Appendix C). The distance between ground truth partitions and partitions extracted from the six predefined time windows is shown for a range of randomization parameters (different percentage ratios for the manipulation of intra- and inter-module edge weights) in Fig.C.1. We found that the given ground truth partitions were completely recovered $(\mathrm{VI}=0)$ from the synthetic network sequences by both extraction approaches for a wide range of randomization parameters and large amounts of introduced "interaction noise". Thereby, the recoverability of the partition corresponding to the sixth time window, which consists of one large and two smaller modules, is particularly sensitive to the amount of randomization. The consensus partitions extracted from network sequences that were simulated with the maximum amount of edge weight randomization that still allowed to reliably identify the ground truth module structure (percentage ratio $40 / 30$, which means $40 \%$ of intra-module edge weight decreases and $30 \%$ of inter-module edge weight increases) are shown in Figs. 3. b) (approach $\# 1$ ) and 3(c) (approach \#2). Due to the large amount of introduced strong inter-module interactions and the resulting weakened module structure quality (Appendix Fig.B.1(b)), minor discrepancies in extracted module affiliations of some nodes in the 2nd $(\mathrm{VI}=0.105)$ and 4 th $(\mathrm{VI}=0.056)$ time windows were revealed for extraction approach \#2. In contrast, for the same level of randomization, the ground truth module structure contained within time windows can still be fully recovered using extraction 

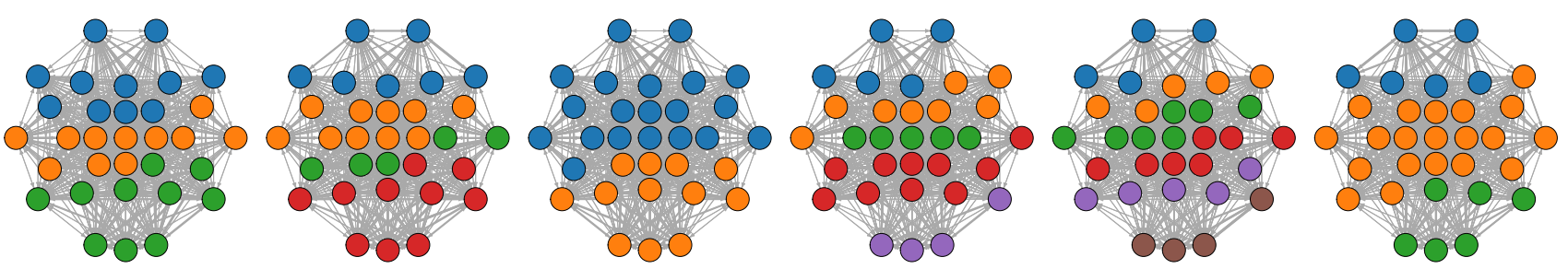

(a)
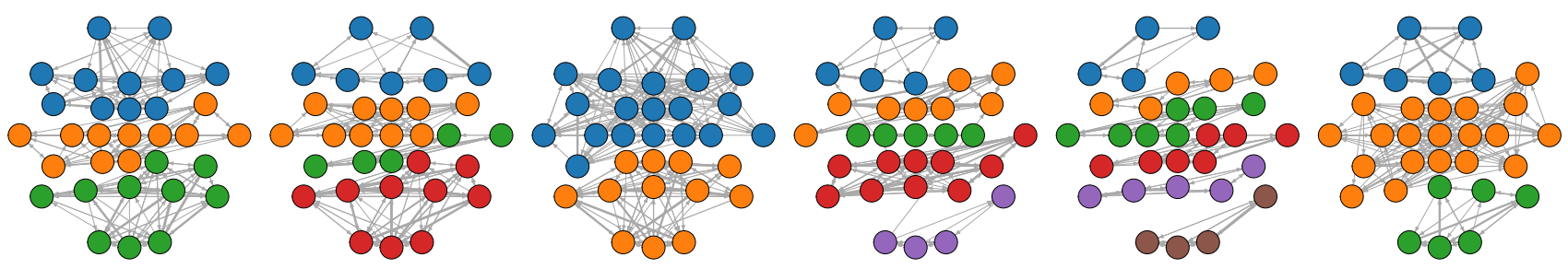

(b)
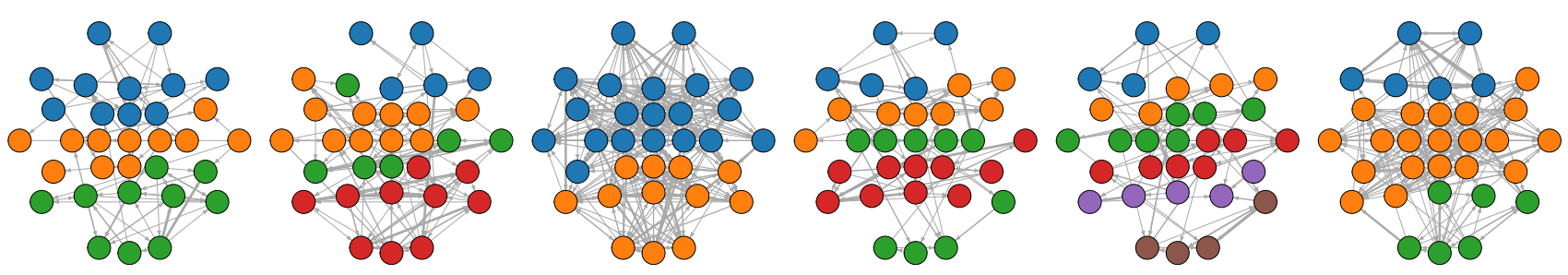

(c)
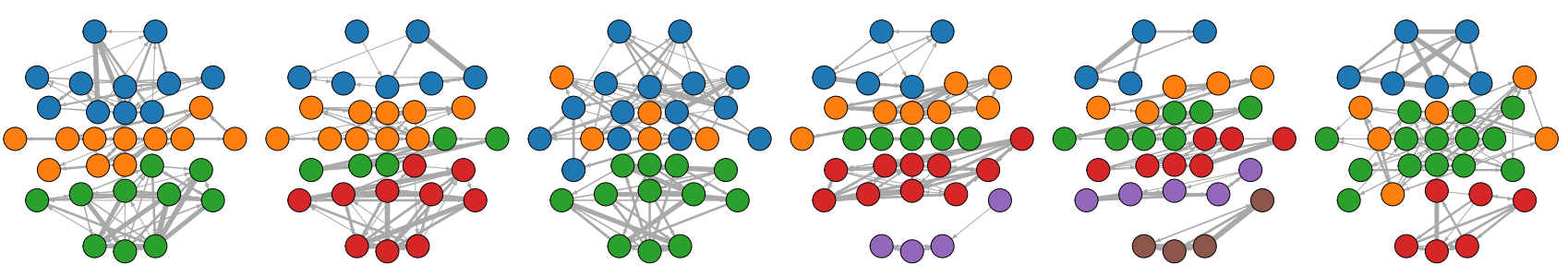

(d)

Fig. 3. Module tracking results in a simulated network sequence. The spatial location of temporal module structure changes is shown in the EEG electrode scheme network layouts. The analyzed network sequence was generated by decreasing the weights of $40 \%$ of intra-module edges and increasing the weights of $30 \%$ of inter-module edges of the initial networks. (a) The ground truth partitions of the initial networks. (b) Consensus partition obtained using approach \#1 (80th percentile). Using standard local thresholding with approach \#1-local (80th percentile of the edge weight distribution of each consensus network) yields an identical module structure on the basis of differently thresholded consensus networks (not illustrated). (c) Consensus partition obtained using approach \#2 (for each time window, the edges of the soft thresholded network that corresponds to the point on the time grid are shown). (d) Consensus partition obtained using the ECO thresholding approach.

approach \#1 (with global multiple thresholding), as well as with extraction approach \#1 with a standard local multiple thresholding step, i.e. \#1-local (see Fig. 3(b)). Using local thresholds, the quality of detected modules for simulated networks is as good as the ones for the global threshold up to the 90th percentile of the edge weights. Thus, the evaluation by simulation cannot fully discriminate between both thresholding methods used for module tracking approach \#1. However, the resulting consensus 
networks of each considered time window are different for both thresholding approaches with regard to the presence and absence of weighted edges.

If the edge weight structure does not show a strong time evolution across time windows and remains homogeneous throughout the network sequence, the proposed global multiple thresholding strategy of module tracking approach \#1 could be replaced with standard local multiple thresholding, where only the edge weights of the consensus network of each respective time window are considered for the computation of thresholds. Note that the resulting thresholded consesus networks most likely will have a different topology.

The module structure identified in networks thresholded by the ECO approach is almost identical to the ground truth (Fig. [3(d)), however for the simulated time windows 3 and 6 , the VI values are consistently larger (Appendix Fig. C.1(c)).

In summary, it can be seen that once meaningful time windows are identified, our approaches and the way they aggregate interaction information within each time window are able to identify, enhance and combine the module structure of its corresponding networks, despite their conceptual differences.

\subsection{EEG recorded time-varying brain functional network}

In our application study, we investigated temporal changes of the brain's information processing before and during the compensation of a major balance perturbation in the form of module structure reconfigurations that were tracked using our computational framework. The first two time points on the time grid were defined with respect to characteristic dDTF changes in the processed dDTF-TFMs, i.e. during the switch from BSS to BUS, and with respect to an equidistant 1-s-grid definition that was used for the rest of the time points. Thereby, from the EEG epoch lasting $8 \mathrm{~s}$, seven points on the time grid and corresponding centered time windows (consisting of 41 networks, equivalent to $400 \mathrm{~ms}$ ) were preselected: $P_{1}=2 \mathrm{~s}$ (BSS), $P_{2}=3 \mathrm{~s}$ (onset of BUS), $P_{3}=4 \mathrm{~s}$, $P_{4}=5 \mathrm{~s}, P_{5}=6 \mathrm{~s}, P_{6}=7 \mathrm{~s}$ and $P_{7}=7.8 \mathrm{~s}$. The last preselected time step was determined by the chosen width of the data aggregation time window.

The results obtained by using approach \#1, which contrasts locally encountered interaction strengths with the globally obtained edge weight distribution, are shown in Fig. 世(a). Multiple global thresholds, given by edge weight percentiles, were computed based on pooling all 800 network observations. The threshold corresponding to the 95th percentile was chosen with respect to the a priori information that no strong interactions exist during $\mathrm{BSS}, 21$ as the resulting thresholded consensus network at $2 \mathrm{~s}$ has no edges and is completely disconnected. Consequently, its partition consists only of isolated nodes, i.e. it has no modules. Thus, the chosen threshold allows for a visualization of the evolution of interaction strengths and its effects on the module structure at the transition between the two balancing conditions and during balancing on the unstable surface. As expected, after the transition to a more demanding balancing task (3-8s), the general localization and the time-evolution of the modules correspond to the expected neural processing for the balancing task. It is known that balancing tasks are associated with significant activation of the fronto-central and centro-parietal cortical regions. Specifically, when balance tasks became more challenging, a significant increase in cortical theta activity was observed in the aforementioned regions. .30 Therefore, midline electrodes (index $z$ - zero) and their immediate neighbors show brain activity that is associated with balancing tasks. Accordingly, modules around the midline are of special interest. It can be seen that the composition of modules is almost mirror-symmetric with regard to the midline (Fig. U(a)). The consensus network at $3 \mathrm{~s}$, the transition to BUS, shows a module, which covers $\mathrm{CPz}, \mathrm{CP} 1, \mathrm{CP} 2, \mathrm{Pz}(\bigcirc)$, i.e. it is located at central $(C)$, centro-parietal $(\mathrm{CP})$ and parietal $(P)$ electrodes. The single electrode $\mathrm{Cz}$ was also identified as a module (), even though it is connected via an outgoing edge to module (O). This assignment is a result of the specifics of the algorithm of Blondel et al $l^{37}$ (e.g. the order in which nodes are considered) and the definition of the modularity quality function for weighted directed networks, which is optimized by this algorithm. Subsequently, both modules expand to neighboring electrodes and remain relatively stable after the transition period (windows at 5-8 s). The central stable module (O) is of particular interest, because Mierau et al ${ }^{21}$ argued that the existence of this module might indicate that the primary motor cortex (M1) could be involved in the compensation 

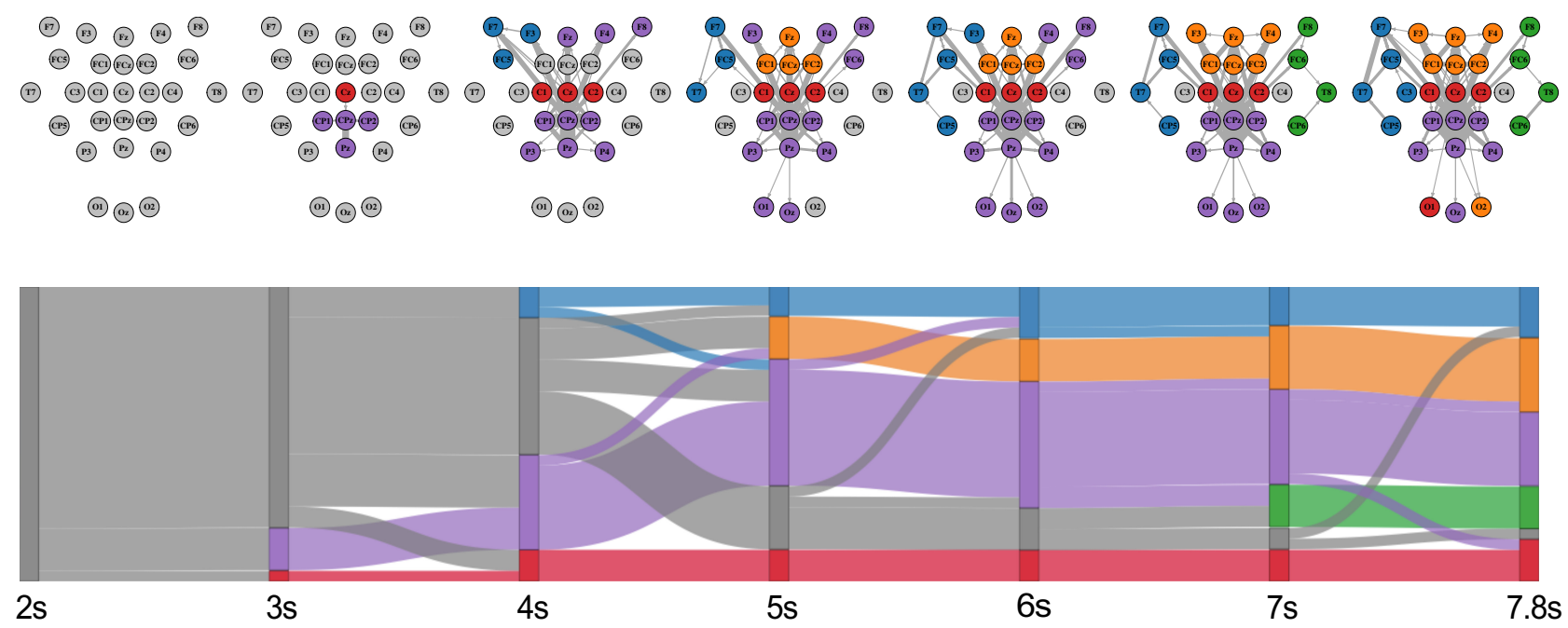

(a) Global threshold (95th percentile)
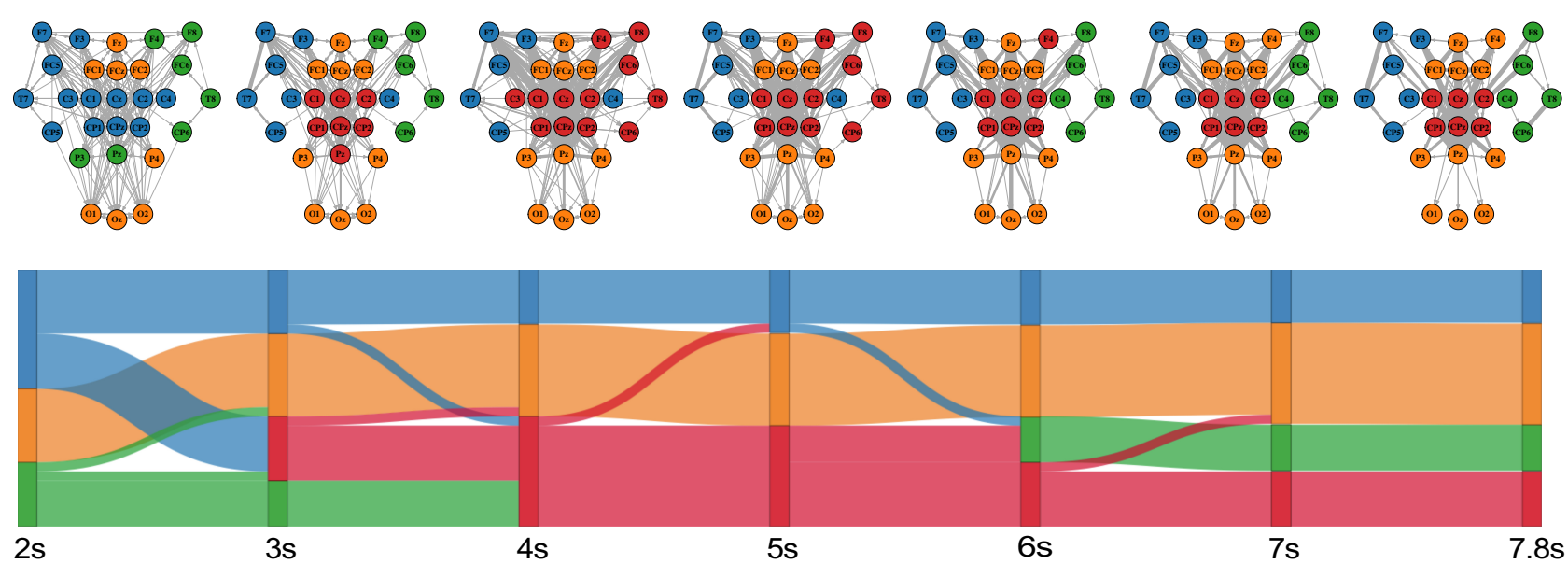

(b) Pareto optimal thresholds

Fig. 4. Dynamic module structure of the theta band networks. The spatial location of module structure changes is shown in the network plots according to the EEG electrode layout, whereas the dynamics, i.e. the flow of node membership, is shown in the Sankey diagrams. Thus, changes of information processing in the brain can be identified from its spatiotemporal module structure changes. (a) Results for the global threshold (95th percentile, consensus networks). (b) Results for the Pareto optimal thresholds (with consensus clustering). For each time window, the edges of the soft thresholded network that corresponds to the point on the time grid are shown.

of the balance perturbation. Furthermore, an additional central module (O) appears in the consensus network at $5 \mathrm{~s}$. This module is formed by the electrodes FCz, FC1, FC2 (fronto-central) and Fz (frontal, midline) and expands to F3, F4 at 7 s, i.e. it is possibly related to the supplementary motor area (medial part) and the premotor area (lateral part, area extrapyramidales) and remains stable thereafter. Associated functions are, for example, motor sequencing and planning as well as movement initiation and inhibition. 54 The corresponding Sankey diagram illustrates the ongoing expansion of the midline modules from $3-5 \mathrm{~s}$, as well as their stability from 5-7 s. A left lateral module (O), reaching from F7 to CP5 shows also an ongoing expansion. In contrast, a right lateral module $(\bigcirc)$ occurs later from $7-7.8 \mathrm{~s}$.

The comparison with the partitions of the consensus networks thresholded with an individual 
threshold (\#1-local) given by the 95th percentile allows the following conclusions (not illustrated): (a) Our approach \#1 shows the dynamics of three continuously expanding modules, which are symmetrically located around the nodes of the midline and two lateral modules. This dynamic is related to the increasing interaction strength, as well as to the increase in the number of interactions. Showing such dynamics was the aim of this thresholding approach.

(b) The approach \#1-local does not show module structure dynamics developing in such a way, because the computation of the threshold does not consider global information about interaction strengths, i.e. it computes thresholds only with respect to the networks within each local time window. The modules around the midline can be observed. In the 6th network, the red module is merged with the green one. The number of disconnected nodes does not decrease over time as observed for approach \#1. The fronto-central module (Fz-FCz) can be considered as being instable. (c) By decreasing the local threshold, the number of disconnected nodes becomes clearly reduced and the module structure changes little over time. The fronto-central module $(\mathrm{Fz}-\mathrm{FCz})$ becomes more stable.

The results obtained by using approach \#2, which exploits local information about the connectivity structure by using thresholds that are Pareto optimal with respect to the module structure quality, are shown in Fig. 4(b). It can be seen that the most distinct changes of the midline modules are associated with the transition from BSS to BUS, i.e. with the transition to a more complex balancing task at $3 \mathrm{~s}$ and $4 \mathrm{~s}$. Thereafter, the consensus partitions remain relatively stable and are similar to those of the consensus networks of approach \#1 at $7 \mathrm{~s}$ and $7.8 \mathrm{~s}$, with the right lateral module $(\bigcirc)$ at $2 \mathrm{~s}$ and $3 \mathrm{~s}$ reappearing at $6 \mathrm{~s}$. The main feature that stands out is the stability of the central module and of the modules, which are associated with the temporal lobes of the cortex ( and ). Contrary to the module structure reorganization uncovered by approach \#1, the midline electrodes $\mathrm{Cz}$ and $\mathrm{CPz}$ are always members of the same module (O). Except for the consensus partition at $3 \mathrm{~s}$, they are always associated with a module that is different from the one of $\mathrm{Pz}$ (mostly ). For approach \#1, we found that $\mathrm{CPz}$ and $\mathrm{Pz}$ always cluster together (O) and are never in the same module as $\mathrm{Cz}(\mathbf{)}$. The node flows corresponding to the dynamics described above are shown in the Sankey diagram, which reveals change points in the module structure reorganization and periods associated with stability.

The comparison of the ECO-based thresholds with our approach \#2 yields the following results: (a) Approach \#2 shows a strong change of the module structure after BUS onset (2nd consensus partition) and remains almost stable during BUS. Two modules around the midline (three modules for \#1, 95th percentile) are obtained, where the fronto-centralparietal-occipital module (O) and the lateral modules cover the central module $(\mathrm{Cz}-\mathrm{CPz})$. The results of approach \#2 are similar to those of the \#1local approach (75th percentile). (b) Approach ECO shows a more unstable module structure in comparison to \#2, with changing numbers of disconnected nodes and where the module structure around the midline also changes after BUS onset from 2 to

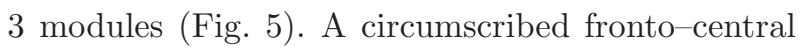
module is revealed.

The global threshold computation using pooled edge weights of all available networks leads to an identified module structure evolution that is associated with the temporal evolution of interaction strengths. In doing so, deviations from this overall information on interaction strengths are highlighted. By contrast, Pareto optimized networkspecific thresholds take the temporal reconfiguration of connectivity structure at each considered time step into account. Therefore, by using different threshold

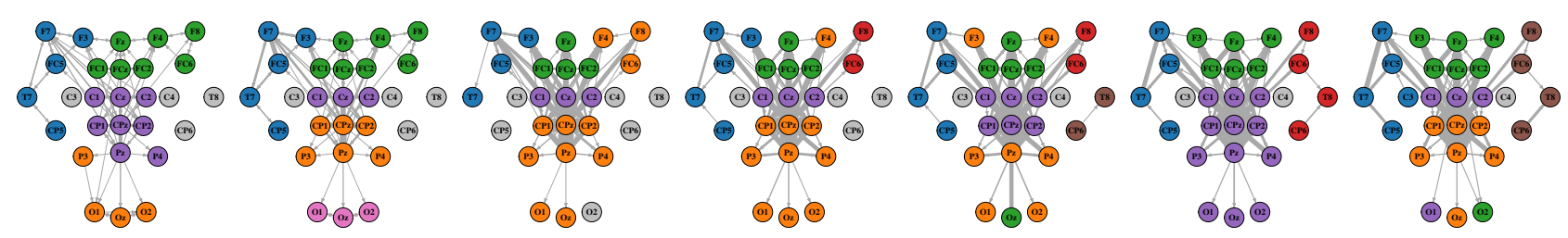

Fig. 5. Dynamic module structure of the theta band networks identified using the ECO threshold approach. The spatial location of module structure changes is shown in the network plots according to the EEG electrode layout. 
definitions, different complementary perspectives on the module structure dynamics are given. Our module tracking framework can be augmented with thresholding procedures other than the ones we proposed for the analysis of EEG-derived time-varying brain functional networks. These thresholding procedures, too, would allow to incorporate a specific view on the temporal evolution of the network structure. In particular, ECO thresholds would shift the analysis more towards information transmission and processing properties of the investigated time-varying network.

In summary, an analysis of the results for the EEG data shows that module tracking approaches \#1 (global threshold), \#2 (Pareto threshold) and ECO are in better agreement with each other, as compared to approach \#1-local, which yields deviating results.

\section{Discussion}

We introduced a general computational framework for the extraction and tracking of temporal network module structure reorganization in timevarying weighted directed edge-complete networks. This framework fills a methodological gap. With regard to the module tracking framework itself, we are not aware of any other method that is able to deal with the high temporal variance of node module affiliations that is typical for EEG-derived functional networks. Due to a lack of available approaches for time-varying module structure analysis, neuroscience research is currently not yet emphasizing such analyses of brain networks and the insights that can be obtained from them. Despite our focus on the study of time-varying brain functional networks, which reflect the evolution of functional interaction structure during neural processing, the presented framework can also be used to identify and analyze the changing module structure in networks from different domains. Moreover, generalizations of the framework to sequences of unweighted and/or undirected networks are straightforward.

For weighted networks, whose edges are not given by measurements, but by estimations or predictions of interactions between represented entities, e.g. brain regions, the thresholding and removal of low weight edges is an integral processing step in the network analysis pipeline to yield edge noncomplete networks and to emphasize key features of network topology $\sqrt[319155]{ }$ Heuristic and statistical thresholding approaches are controversially discussed ${ }^{16}$ and have to be applied carefully as they are prone to introduce serious biases into the resulting network structure $\frac{18}{18}$ The common situation of having to screen various predefined edge weight thresholds and to subsequently analyze the resulting thresholded networks in an exploratory fashior $\sqrt{35136}$ is improved with our proposed algorithm for computing a specific single Pareto optimal threshold for each network (approach \#2). Using multicriteria optimization, different objective functions that quantify certain structural characteristics of the thresholded network can be simultaneously optimized. The Pareto optimal thresholds computed by our approach are objective and have a clear meaning with respect to the obtained thresholded network structure - these thresholds guarantee that the underlying weighted (or binary) network module structure is most pronounced with respect to the evaluated module structure characteristics. We note that a similar approach based on multicriteria optimization can be applied to each individual network to find the module detection algorithm that yields the highest quality network partitions. This, however, was not done in this work. Recently, Fallani et al. suggested a method 19 to compute edge weight thresholds by pursuing an objective similar to our method, i.e. by seeking to optimize higher level structural properties of the thresholded network according to defined quality functions. In their case, a single quality function accounts for the trade-off between local and global efficiency of the network ${ }^{34}$ and its wiring costs, which are given by the connection density. Like our method, the weakness of their approach is the consequent removal of the weakest interactions from the network, despite the known "strength of weak ties", 56,58 the role of "bridge" edges 59 and the fact that even edges with low weights can be statistically significant. We used the ECO threshold as an alternative for our Pareto optimal thresholds. In this way, we have given a contribution to a time-variant extension of Fallani's approach, too. In this regard he stated: "In neuroscience, functional brain connectivity exhibits rich temporal dynamics that are fundamental for human cognition and complex behavior. Further studies should aim to elucidate if and how brain network differences highlighted by ECO change over time." $[19$ For our data, we found 
that the ECO approach is able to reproduce principal features of the module structure identified with approach \#2 (and to some degree also with approach \#1), despite yielding module structures consisting of disconnected nodes. Future research should aim at extending such concepts to compute thresholds for separate substructures of the network, or even for single edges.

Modules in partitions extracted at different times have to be structurally related to each other to track their reorganization, which constitutes the end result of the processing steps of the framework. For it, we proposed an algorithm for globally matching modules at optimal cost among the consensus partitions, which inherently accounts for the module structure evolution. The parameter $\theta$ has a big influence on the matching results, as a trade-off between eliminating poorly fitting matches and an excessively colored, diffuse module structure has to be made, in particular if large network sequences are considered. In addition to our global module matching approach, a local module matching algorithm was implemented and applied to the presented consensus network partitions. It is based on the heuristic dynamic community tracking approach proposed by Greene et al. 60 which accounts for all nodes that ever belonged to the so-called dynamic communities. The matching strategy of the local algorithm exploits the information about module similarity contained in the time line of dynamic communities, which are generated by matching the so-called step communities identified at each point in time to the last observation of all dynamic communities. In contrast, for our purposes, step communities identified at consecutive points in time also have to be matched among each other. For each considered point in time, the module matching is performed in a two-step process. First, dynamic community time lines are adjusted or created from scratch in a mostly local fashion by independently matching each newly identified step community to the last observation of all dynamic communities as described in their paper 60 In the second step, the information about step community assignments to dynamic communities is used for obtaining good local module matches. The central algorithmic idea is to compare for each dynamic community $D_{i}$ the node set of the currently matched step community and the node set of the previously matched step community to the time line of all other dynamic communities.
Thereby, it is counted how often the last matched node set of $D_{i}$ is encountered as the last matched node set of any other dynamic community. Likewise, it is counted how often the second last matched node set of $D_{i}$ occurs as second last matched node set in any other dynamic community. A proper module matching that accounts for "life cycle" events in the evolution of module structure can be inferred with these two information. In our application, both algorithms yielded similar results and only those of the global approach are presented.

We applied our framework for the analysis of an EEG-derived time-varying brain functional network that reflects the changing functional interaction structure of a group of healthy subjects before and during the neural processing of a balance perturbation. Balance control, whose underlying neurophysiological mechanisms are still not completely clarified, is a fundamental component of every day human motor activities such as standing or walking and its impairment is associated with an increased risk of falling. ${ }^{21}$ It requires a complex interplay within and between the sensory and the motor systems, which makes time-variant connectivity analysis particularly suitable for its study. The high-dimensional multichannel recording of brain activity during the balance control experiments (one-legged balancing on a stable and unstable surface) is only possible with EEG, where motion-related and other artifacts must be rejected or their influence on the connectivity results must be minimized. The EEG data and the tvMVAR modeling results used in this study were thoroughly inspected with regard to artefactual influences. ${ }^{21}$ However, the interpretation must be done carefully because effects of volume conduction can influence the results of the DTF analysis. $\stackrel{28}{ }$ Therefore, the results of our EEG application are preliminary. The debate concerning volume conduction gave the impetus for the development of evaluation and benchmarking strategies, which will be helpful to improve connectivity analyses ${ }^{21}$ A second reason why the results of the EEG study are preliminary is because the real neurophysiological relevance of our application results will only become visible by using our framework for the analysis of the module structure dynamics of both frequency band networks, i.e. the theta- and the alpha-band network, as well as of their temporal coordination. This will be the subject of further research. 
Thereby, the choice of the investigated time grid and the width of time windows affects the volatility and quality of tracked consensus partitions. Clearly, if the widths of time windows does not match the natural time scale of the module structure evolution, certain meaningful features of the module structure reorganization would be missed by our framework. Predefined points on the time grid $P_{g}$ do not need to be equidistant and the width of time windows can vary so that they are better adapted to the rate of network topology changes. A tuning of these parameters requires prior knowledge or exploratory data analysis. In the context of EEG-based brain functional networks, the results of the time-frequency analysis constitute a reliable way for extracting information about important events in the temporal evolution of recorded brain activity (Appendix E).

The results are also strongly affected by the choice of the algorithm for the identification of network modules. Nowadays, a great wealth of methods for module identification with unique algorithmic features is available ${ }^{10} 12$ Since these algorithms exploit and interpret structural information inherent to the network data differently, identified network partitions are not necessarily unique, which means that different partitions of similar quality and equal legitimacy might exist. Adding to this problem is the fact that classical module structure quality functions, like modularity ${ }^{41}$ typically lack a clear global maximum and allow for an exponential number of diverse, high-scoring alternative network partitions. ${ }^{[6]}$

An interesting avenue for further research will be the automation of the time grid definition based on the topology of networks in different parts of the network sequence. One way this might be achieved is by utilizing perturbation analysis $\frac{62}{64}$ to assess changes of consensus module structure stability depending on the position and width of the aggregation time window.

Furthermore, to verify the robustness and generality of our proposed module tracking framework, it should be applied to time-varying network data from other experimental tasks and conditions. With regard to balance control, one might think about tracking the module structure associated with the "surprise" effect of an unpredictable balance perturbation as well as changes occurring as a result of physical and/or mental fatigue.
Concluding the paper, we have demonstrated that the extraction and tracking of module structure reorganization in time-varying brain functional networks offers new perspectives on the chronology of brain function and can reveal information about the temporal redistribution of strong interactions that remain absent from the analysis of stationary network representations of functional connectivity. It can be expected that the application of our framework to time-varying networks from other domains will reveal similar new insights.

\section{Acknowledgments}

This work was supported by the DFG grants Wi 1166/16-1 (H. Witte) and INST 229/2-1 FUGG (A. Mierau).

\section{Appendix A. EEG Electrode Layout}

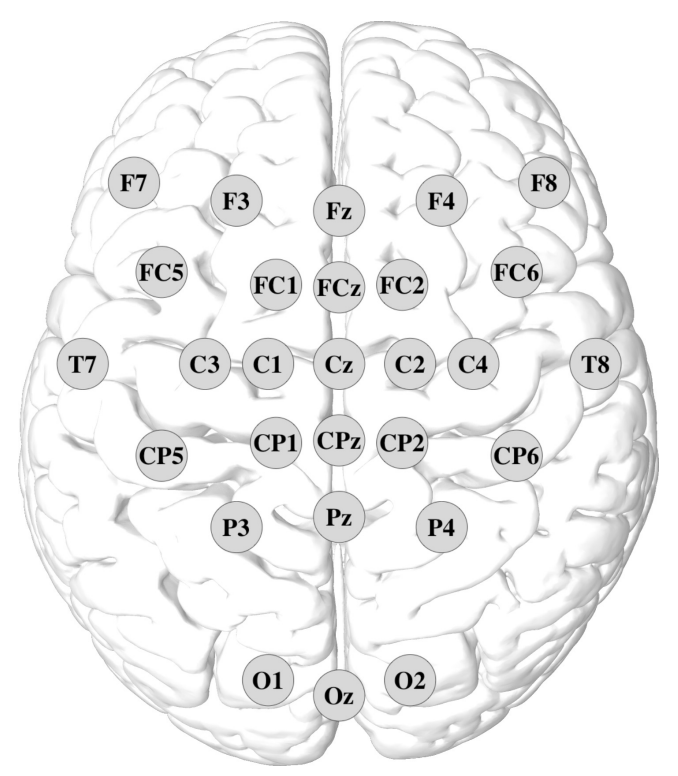

Fig. A.1. EEG electrode layout considered for the neuroscience study of this paper. The shown EEG electrode positions were represented as nodes in the time-varying brain functional network that represents the group averaged neural processing during the balancing task.

\section{Appendix B. Simulation of Time-Varying Weighted Edge-Complete Networks with Ground Truth Module Structures}

In this section we describe the procedure to generate the time-varying synthetic network data. It 
consists of a sequence of weighted edge-complete networks, which is divided into several segments that each correspond to a time window in which the underlying module structure is relatively stable and remains mostly unchanged despite varying network topologies. The entire network sequence is generated by a series of successive computational steps. First, the number of time windows is specified and for each time window, an initial weighted directed edgecomplete network with corresponding unsymmetrical edge weight matrix is simulated. Subsequently, for each of these initial networks, a set of network instances with randomly perturbed edge weights is generated using the method described below. These perturbed and randomized networks constitute the network observations of the time window to which the initial network corresponds. The perturbation of the edge weight structure weakens the underlying weighted module structure by introducing "interaction noise". These simulations are described in the following.

\section{B.1. Simulation of a weighted network with known module structure}

The simulation consists of two steps. At first, the adjacency matrix of the underlying binary network that contains the information on edge positions is obtained by means of using an algorithm similar to the one we described previously. 16.65 Thereby, properties of the module structure are controlled by probabilities of intra- and inter-module edges,
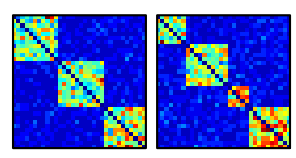
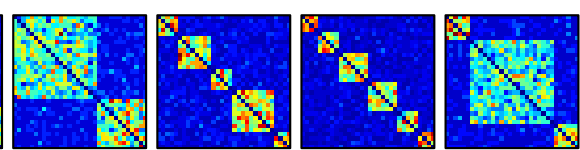

(a)
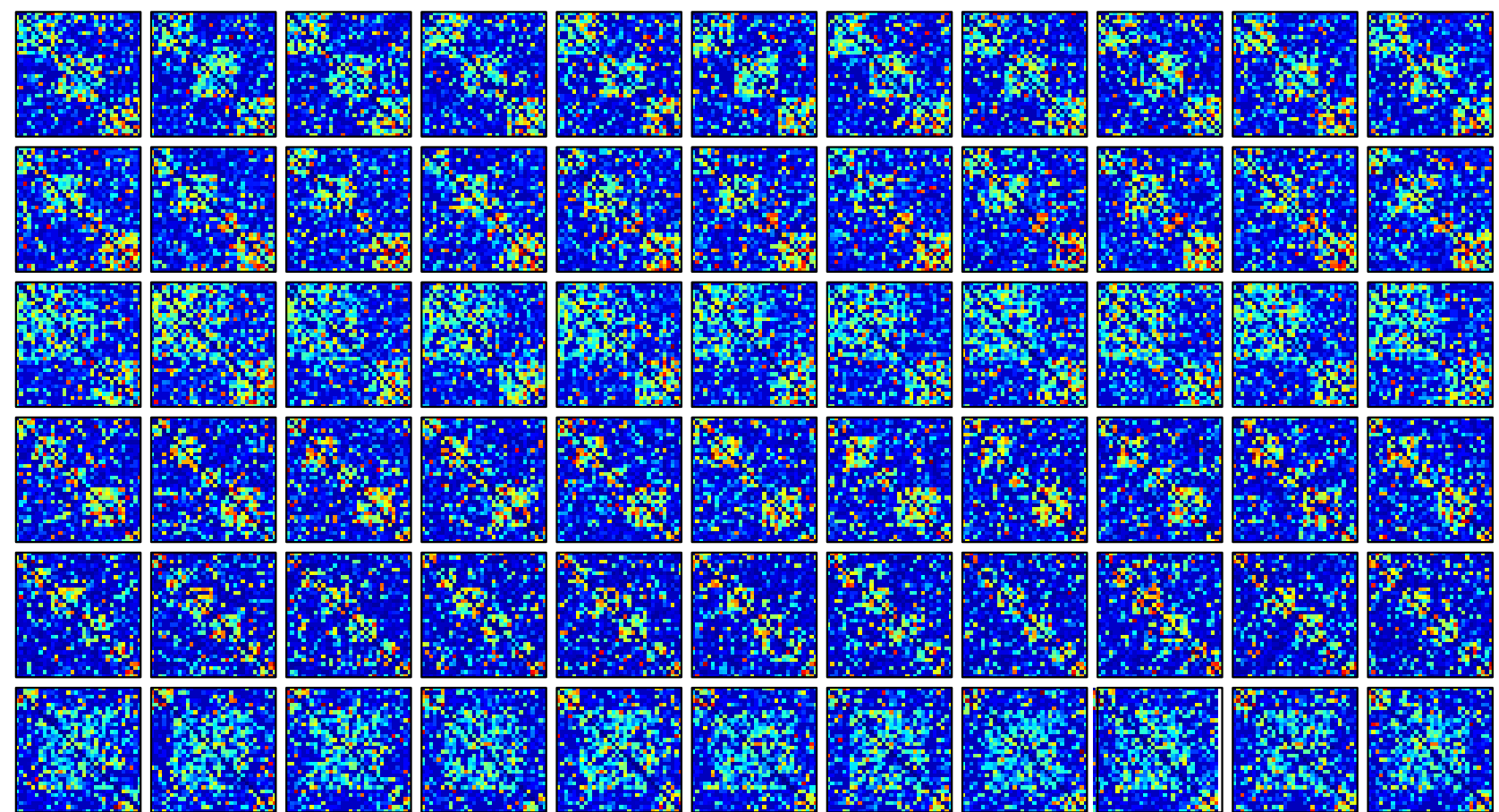

(b)

Fig. B.1. Edge weight matrices for the six simulated initial networks with ground truth module structure and the networks that are based on them. (a) Weighted directed edge-complete initial ground truth networks, whose modules are apparent in the form of a block-diagonal structure of the associated edge weight matrices. (b) Sequences of 11 randomly altered networks are derived from each initially simulated network by decreasing the weights of $40 \%$ of intra-module edges and increasing the weights of $30 \%$ of inter-module edges. 
user-defined module memberships of nodes, specified constraints on minimum intra-module in- and out-degrees and constraints on maximum intermodule in- and out-degrees. For the testing of our computational framework, we need to simulate a sequence of weighted edge-complete networks, thus the underlying binary networks also have to be edgecomplete. Given an appropriate assignment of simulation parameters, edge-complete binary networks could be generated with this algorithm. However, all entries of the corresponding adjacency matrices, apart from the main diagonal, can be directly and trivially set to 1 without requiring any further computational effort for simulating them. The second step of the simulation entails a probabilistic aggregation of edge weights to obtain an edge weight matrix from an adjacency matrix. To this end, a probability for intra-module and inter-module edge weight aggregation has to be specified, together with the number of aggregation attempts. Thereby, a clear module structure results from high probabilities for intra-module edge weight aggregation and low probabilities for inter-module edge weight aggregation. During each edge weight aggregation attempt, depending on both given aggregation probabilities and the outcome of a probabilistic decision of the algorithm with respect to both kinds of weight aggregations, an intra- and/or an inter-module edge is selected randomly and its weight is increased by one unit. If an intra-module edge is going to be increased, the algorithm randomly picks one module according to user-specified probabilities and selects one of its intra-module edges uniformly at random. The probabilities for picking a module can be tuned to either preferentially increase the strengths of internal interactions of certain modules or to mitigate the effect of

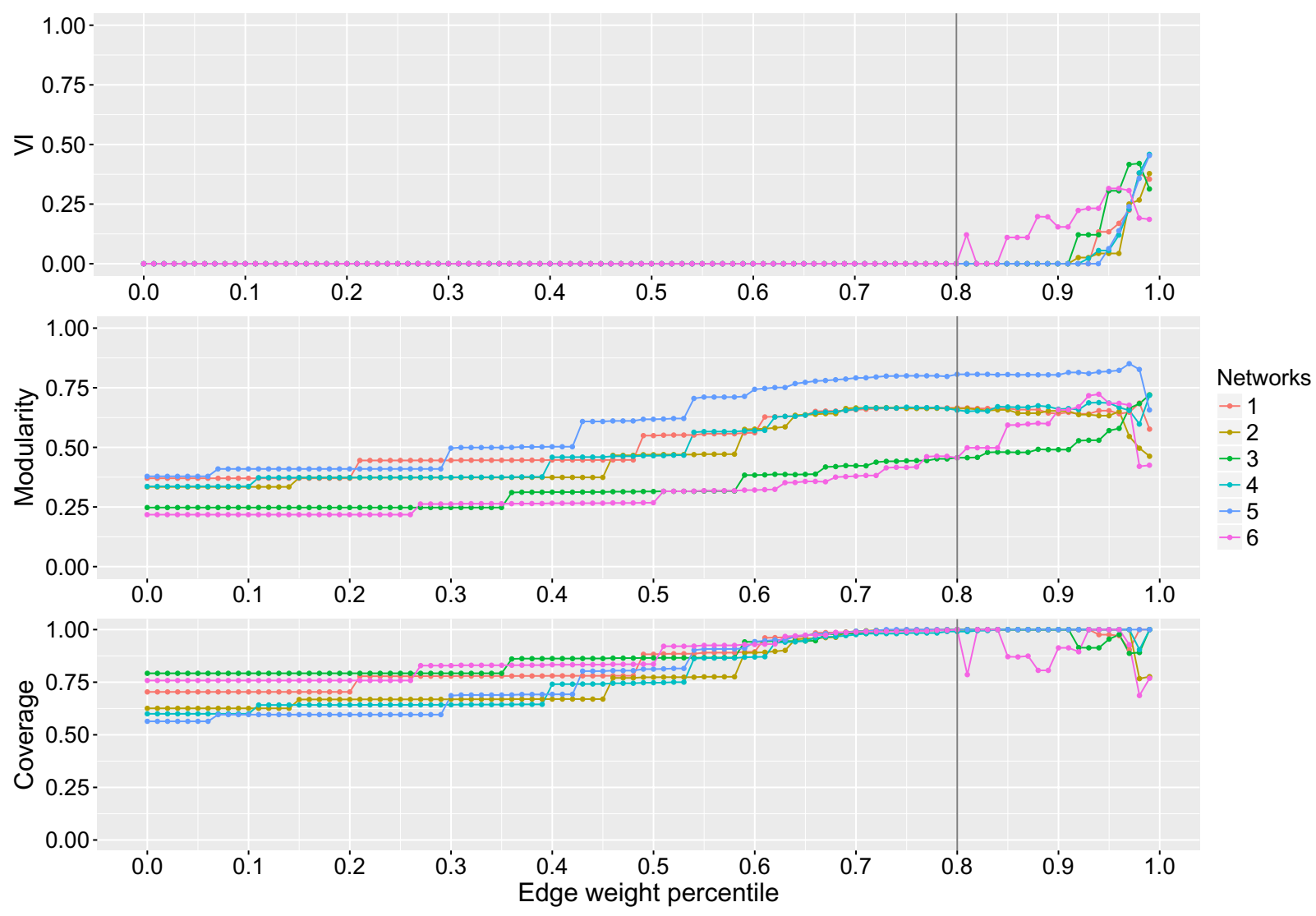

Fig. B.2. Selecting a suitable global edge weight threshold. For a simulated sequence edge complete networks with ground truth partitions, it is possible to select an edge weight percentile as threshold so that consensus partitions extracted from predefined time windows using the threshold have maximum similarity to the corresponding ground truth partitions (minimum VI) in addition to a good structural quality and definiteness. The time-varying network simulated for the randomization parameter percentage ratio 40/30 was analyzed. 
module size on intra-module edge weights. Clearly, in edge-complete networks, the number of intra-module edges depends on the module size, as every node of the module is connected in both directions to every other member node. Given all modules have an equal probability for being selected for intra-module edge weight aggregation, individual intra-module edges of smaller modules would be selected more repeatedly for weight aggregation, resulting in them acquiring stronger edge weights as compared to individual edges inside larger modules. Due to a larger number of edges being situated inside larger modules, individual edges will be selected less frequently and thus will, on average, acquire less weight. By tuning the module selection probabilities accordingly, intra-module edge weights can be distributed homogeneously across modules. If during an edge weight aggregation step, it is decided that an inter-module edge weight should be aggregated (either on its own or in addition to an intra-module edge), a specific inter-module edge is selected uniformly at random from all such edges and its weight is increased by one unit. After all aggregation attempts are performed, the edge weights are rescaled to the $[0,1]$ interval.

\section{B.2. Simulation of networks with randomly perturbed edge weights}

After obtaining an initial weighted directed edgecomplete network with known module structure, the aim is to add predefined levels of edge weight perturbations to generate "noisy" instances of the initial network that make up the time-varying network sequence. This aim is achieved by selectively decreasing the weights of a percentage of intra-module edges and independently increasing the weights of a (possibly different) percentage of inter-module edges. All edge selections are performed uniformly at random. The values that are added to or subtracted from edge weights are drawn from the beta distribution with parameters $\alpha=1, \beta=3$ for edge weight increases (tends to yield lower values) and $\alpha=2, \beta=2$ for edge weight decreases (tends to yield medium values). Whenever edge weight decreases result in an edge weight smaller than or equal to zero, the respective edge weight is set to the minimum edge weight of the initial network on which the perturbations are based. If edge weight increases result in edge weights larger than one, the respective edge weights are set to one. Each edge (intra-module and inter-module) is considered exactly once for a manipulation.

For the testing of the dynamic module structure extraction framework, several synthetic time-varying network sequences were generated for varying edge weight randomization parameter settings.

\section{Appendix C. Selecting a Global Edge Weight Threshold for Approach \#1}

To test the computational framework, we compared the extracted consensus partitions of time windows of several simulated noisy network sequences (generated for different randomization parameter values that result in different amounts of "interaction noise", i.e. edge weight structure perturbations) to the respective known ground truth module structures (Sec. 4.1. Fig. C.1). For the application of the timevarying module structure extraction approach \#1, a percentile of all globally available edge weights has to be specified for the soft thresholding of the consensus networks of the time windows. For the simulation study, it is possible to analyze the effects of threshold levels with respect to the correctness and quality of the obtained consensus network partitions. For this analysis, we considered the synthetic time-varying network generated with randomization parameter percentage ratio $40 / 30-40 \%$ of intra-module edge weights were decreased and 30\% of inter-module edge weights were increased for the generation of single network instances based on their associated initial networks with ground truth module structure. This parameter percentage ratio resulted in the maximum amount of edge weight randomization that still allowed to reliably identify the ground truth module structure. This time-varying network was processed for a wide range of percentile values and the extracted consensus partitions were compared to the ground truth partitions by means of the normalized VI (partition distance) $\stackrel{53}{5}$ while their quality was assessed using the modularity 41 and coverage ${ }^{12[16}$ measures. The results of this analysis are shown in Fig. B.2. We found that a wide range of thresholds with a removal of up to $80 \%$ of all edges does not affect the correctness of extracted consensus partitions with respect to the ground truth. The 80th percentile was chosen as final threshold for approach \#1 for the simulation study, because it is within the range of thresholds where the accuracy of module 


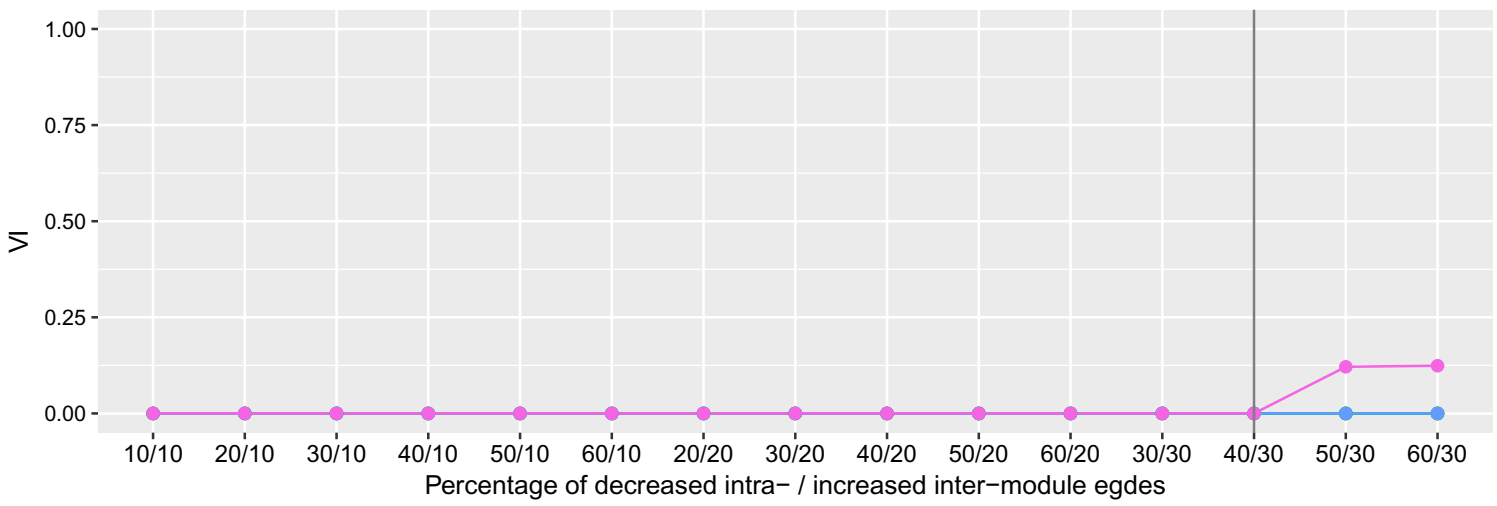

Time window consensus

- 1

$-2$

$-0-3$

$-4$

$-5$

$-6$

(a)

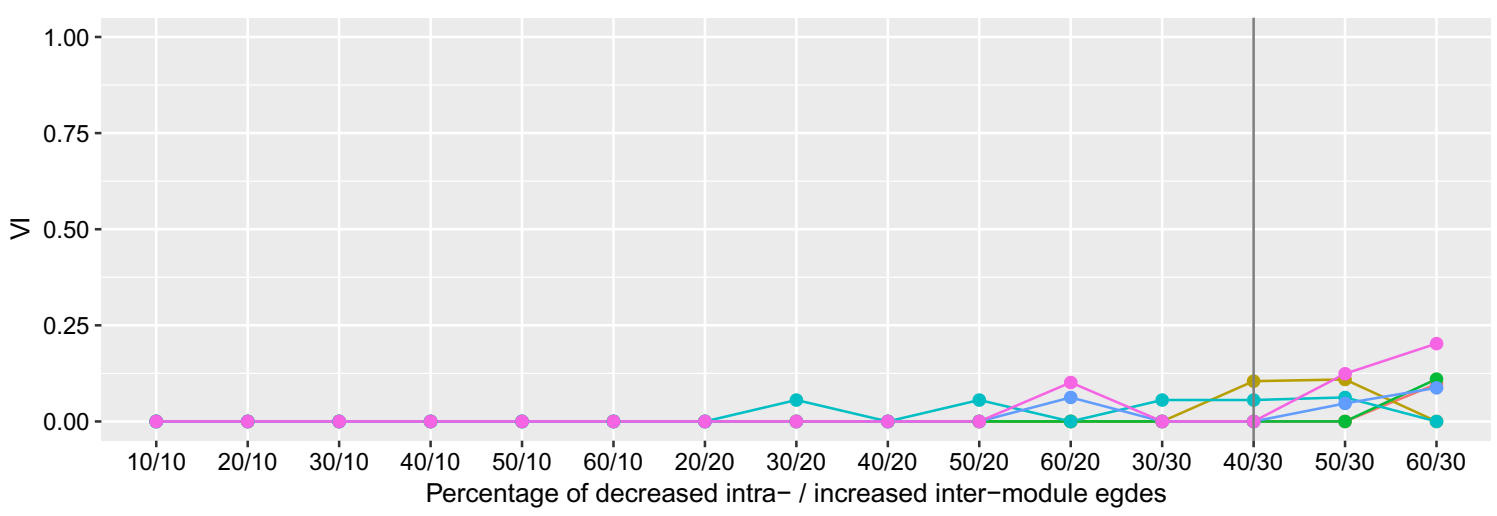

Time window consensus

- 1

$-2$

$-3$

$-4$

- 5

$-0-6$

(b)

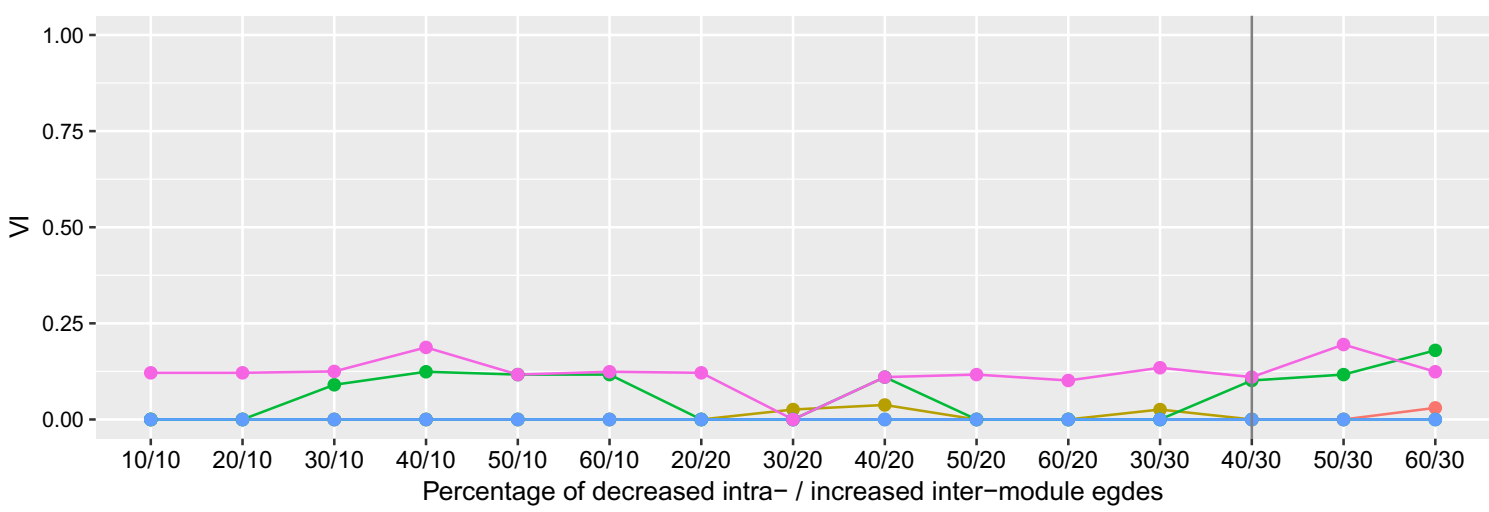

Time window consensus

$-1$

$-2$

$-0-3$

$-04$

$-0$

$-6$

(c)

Fig. C.1. Recoverability of ground truth module structure within each time window of simulated network sequences. For network sequences generated with different randomization levels, the distance (normalized VI (VI) $\underline{53}$ ) between the ground truth partition of each time window and the corresponding extracted partition was quantified. (a) Results for approach \#1 (80th percentile). (b) Results for approach \#2. (c) Results for approach ECO. 
structure extraction is not affected by the soft thresholding and because for it, the modularity and coverage measure attain their maximum values.

\section{Appendix D. Time-Frequency Analysis of the EEG Data}

Time-variant spectral analysis 21 of the EEG data using the matched Gabor transform $\frac{66}{6}$ reveals the presence of strong artifacts caused by the experimental conditions (e.g. "sway-related" artifacts, EOG interferences and body movement artifacts) in the EEG data from electrodes FP1, FP2, P7, P8, T7, T8 (Fig. D.1). The conclusions drawn from these investigations are that interactions which originated from the electrode sites Fp1 and Fp2 should be excluded from an interpretation and those related to the sites $\mathrm{T} 7, \mathrm{~T} 8, \mathrm{P} 7$, and $\mathrm{P} 8$ must be interpreted carefully. However, the influence of artifacts in EEG activity on the central, centro-parietal, parietal and occipital network interactions was found to be only marginal. In particular, the influence of the signals from these six electrodes on the estimation of the tvMVAR parameters of the remaining signals was found to be minor compared to a reduced tvMVAR model that excludes these electrodes. Network visualization using the entire set of electrodes reveals that electrodes Fp1, Fp2, P7 and P8 act solely as sources of interactions. These four electrodes were excluded for the dynamic module tracking since their characteristics had a negative influence on the
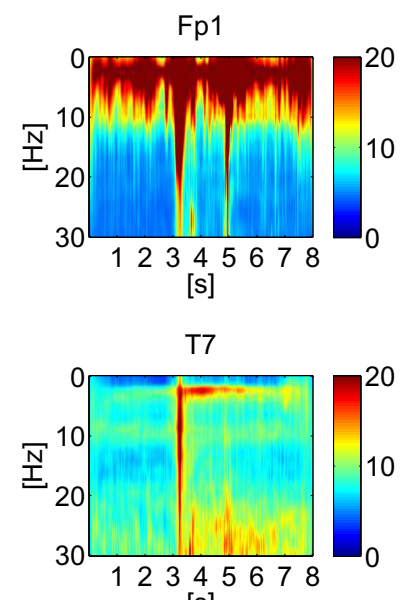

[s]

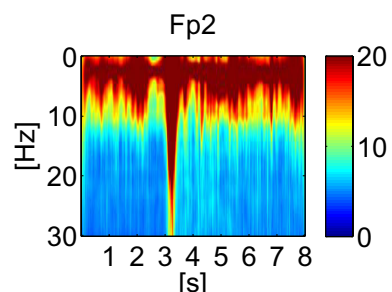

[s]

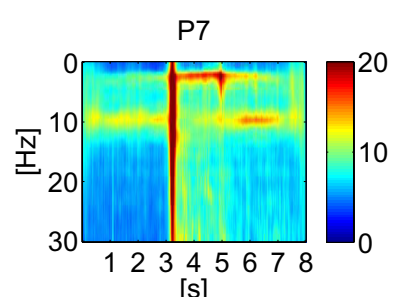

[s]
Fig. D.1. Grand mean TFMs for electrodes Fp1, Fp2, T7 and P7 as examples for artifact-contaminated signals. The platform perturbation onset is at time $3 \mathrm{~s}$. detection of network modules. After the removal of the corresponding nodes, networks of the resulting two network sequences that form the two frequency band-specific dynamic networks consisted of 28 nodes. The selected EEG recording sites used in this study are shown in Fig. A.1

In Fig. D.2 the grand mean time-variant amplitude spectra of the tvMVAR parameters, during the balancing task is represented for electrodes $\mathrm{CPz}$ and O2. These illustrate the main properties of frequency band activity in our data, which are strong theta oscillations at about $6 \mathrm{~Hz}$ (e.g. CPz, Fig. D.2 left) and alpha oscillations at $10 \mathrm{~Hz}$ (e.g. O2, Fig. D.2 right). A statistical comparison of the theta activity before and after the perturbation onset (platform release) was carried out by a confidence tube analysis. In particular, at $\mathrm{CPz}$, the mean amplitude within the theta sub-band increases about $2 \mathrm{~s}$ after the onset (from about $7.5 \mu \mathrm{V}$ to $10 \mu \mathrm{V}$ ). For the electrodes $\mathrm{CPz}$ and $\mathrm{Pz}(5-8 \mathrm{~s})$ as well as $\mathrm{Cz}, \mathrm{C} 1, \mathrm{CP} 1, \mathrm{CP} 2(5-6 \mathrm{~s})$, the mean theta amplitude significantly increases during the balancing task on the unstable platform. For each of these electrodes, no overlap of the confidence tubes (95\% confidence interval) during balancing on a stable or unstable surface could be observed, meaning that there is a statistical significant difference in activity between these two balancing conditions in the theta band. In addition, a strong alpha amplitude suppression ("alpha drop") occurs at the occipital electrodes and, in a weaker form, at the parietal electrodes. The "alpha drop" reaches its maximum about $1 \mathrm{~s}$ after the platform perturbation onset and persists for about $1 \mathrm{~s}$. A statistically significant difference of
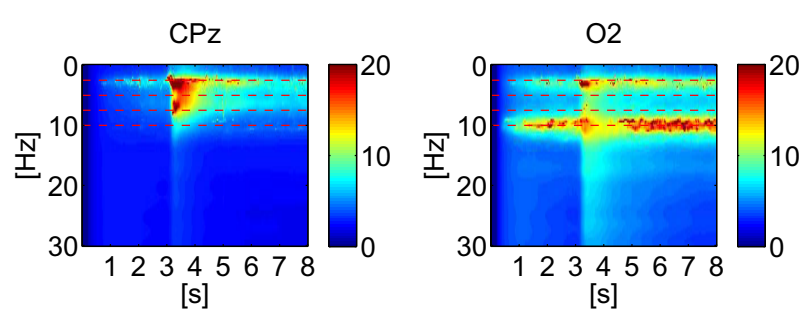

Fig. D.2. (Color online) Selected results of the grand mean time-frequency analysis (amplitude spectrum) for the EEG at $\mathrm{CPz}$ and $\mathrm{O} 2$. The time-variant amplitude spectra are based on our tvMVAR approach. The color coding represents absolute amplitude values $(\mu \mathrm{V})$. The horizontal red dashed lines designate 2.6, 5.2, 7.8 and $10.4 \mathrm{~Hz}$ (possible harmonics of the sway-related interference). The balancing task on an unstable platform starts at $3 \mathrm{~s}$. 
the alpha amplitude between the two balancing tasks after the "alpha drop" was not observed in the confidence tube analysis.

It can also be seen that after the platform perturbation onset, the results of the time-frequency analysis (time-variant amplitude spectra) on the basis of tvMVAR modeling are strongly distorted by movement-related artifacts. This means that also the results of module detection and tracking are affected. As a consequence (cf. Fig. D.2), the results associated with the time interval $3-4 \mathrm{~s}$ should be interpreted with caution.

\section{Appendix E. Time-Frequency Connectivity Analysis of the EEG Data}

As described in the main text of this paper, the timefrequency connectivity analysis of this study was performed by means of fitting multi-trial grand mean EEG data (i.e. for each directed interaction between two different electrodes at each time and frequency: taking the mean over all subjects) using the general linear Kalman filter ${ }^{25}$ to obtain time-variant MVAR model parameters, which are required for the computation of functional interactions by means of the dDTF $\stackrel{27}{27}$ The results of the dDTF can be represented visually by $D \cdot(D-1)$ time-frequency maps (dDTFTFMs), where $D$ is the number of electrodes. Each dDTF-TFM represents the strength of a directed interaction among two different electrodes at specific frequencies and points in time (dDTF-TFM: $x$ axis represents time, $y$-axis represents frequency, $z$ axis (color) represents dDTF values, i.e. interaction strengths). As an advantage of the dDTF compared to other connectivity measures, e.g. partial directed coherence $(\mathrm{PDC})^{67}$ the resulting dDTF-TFMs have a good frequency resolution so that frequency bands can be separated with high accuracy.

In summary, it can be stated that using the dDTF for the computation of functional interactions together with the applied post-processing steps, i.e. averaging of interactions over subjects and extracting the median value at each time step from the averaged dDTF-TFMs for a frequency (sub-)band of interest, results in a group- and frequency band-specific dynamic functional brain network with a topology that accurately describes the effect of certain oscillations on the connectivity structure between recorded time series.

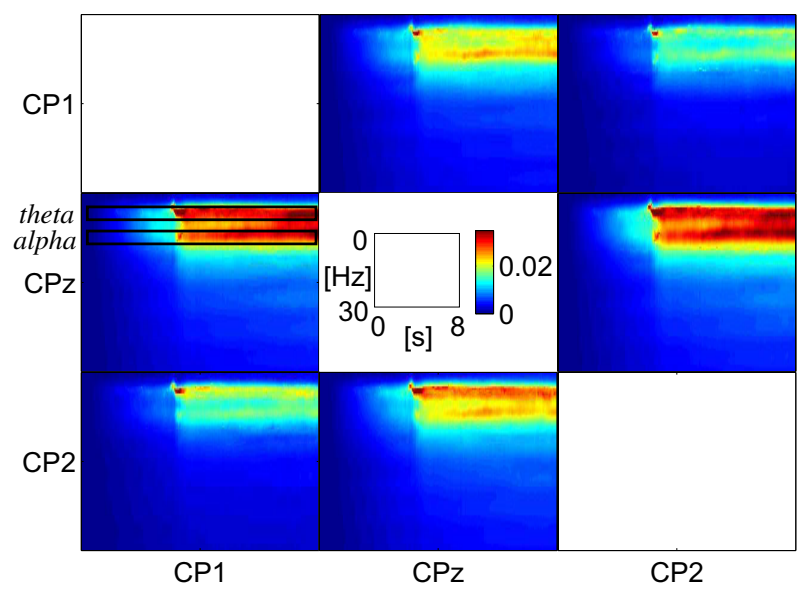

Fig. E.1. Example of grand mean dDTF time-frequency connectivity maps for electrodes $\mathrm{CP} 1, \mathrm{CPz}$ and $\mathrm{CP} 2$. Single dDTF-TFMs are ordered so that the direction of interaction between depicted pairs of electrodes is read from rows to columns. The theta and alpha frequency bands are highlighted by the two rectangles. It is clear that the theta and alpha networks can be seen as distinct networks. The sudden amplitude increase in the dDTFTFMs corresponds to the switch from BSS to BUS.

We were interested in the neuronal activity reflected in the theta and alpha band, whose separation is apparent in the time-frequency connectivity maps obtained with the dDTF ${ }^{21}$ An example of the connectivity results for a subset of electrodes (CP1, $\mathrm{CPz}$ and $\mathrm{CP} 2)$ is shown in Fig. E.1 It can be seen that the switch from BSS to BUS is marked by a sudden increase in amplitude, i.e. interaction strength. During the balancing task on an unstable surface, the amplitude is still increasing, but the variation is much lower and not as pronounced. This observation allowed us to consider larger segments (wider time windows) for the computation of the consensus network structure and consensus clustering (see Fig. 2).

\section{Appendix F. Time Evolution of Modules}

As networks change over time, so do the network modules they contain. The "life cycle" of modules ${ }^{\text {a }}$

\footnotetext{
$\overline{\mathrm{a} A}$ more fine-grained analysis, which adopts a nodecentric perspective to consider and track the dynamic interactions between nodes and the influence individual nodes have on dynamic network modules, is also possible. 68
} 
is given by formation events, growth processes, contraction processes, mergers, splitting events, continuation events and dissolution events $\frac{69}{} \mathrm{~A}$ module formation event is being observed if a module $k$ emerged at time $n, \mathcal{M}_{k}^{n}$, for which no corresponding (similar) module exists at time $n-1$ (or, if also nonsubsequent time steps are considered, at any previous time step). During module growth or contraction, the module gains or loses a considerable amount of member nodes. Mergers of modules occur between successive time steps if at least a minimum percentage $\mathcal{K}$ of nodes of $k$ previously separated modules $\mathcal{M}_{1}^{n-1}, \ldots, \mathcal{M}_{k}^{n-1}$ have aggregated and are fused together to form a new module $\mathcal{M}_{m}^{n}\left[{ }^{68}\right.$ In a splitting event, the opposite situation appears, since from on time step to another, a module $\mathcal{M}_{m}^{n-1}$ is fragmenting into different smaller detached modules $\mathcal{M}_{1}^{n}, \ldots, \mathcal{M}_{k}^{n}$ if a sufficiently large percentage of its nodes are members of the new separated modules. Module splits signify that a considerable fraction of interactions between co-member nodes is lost between two successive time steps. A continuation event is present if a module observed at one time step $n-1$ remains largely unchanged at the following time step $n$ with respect to its node composition $\left(\mathcal{M}_{k}^{n-1}=\mathcal{M}_{k}^{n}, \mathcal{V}_{\mathcal{M}_{k}^{n-1}} \sim \mathcal{V}_{\mathcal{M}_{k}^{n}}\right)$ so that both observations of the module can be trivially matched one-to-one. A module dissolution event occurs if a given module has not been observed from one time step $n-1$ to another time step $n$ (or, in a different notion, if also nonsubsequent time steps are considered, for at least $d$ consecutive time steps ${ }^{60}$ ). If also nonsubsequent time points are considered for the module tracking analysis, it is possible to classify modules as intermittent if they are not found during all observed time points. To discern the intermittence of module existence from a dissolution event, a maximum number of time steps in which an intermittent module is required to reoccur has to be defined.

\section{Appendix G. Assignment Problem}

An assignment problem is given by a set $W$ ("worker"), whose elements are assigned to a set $T$ ("tasks"), with $|W| \leq|T|$ and a $|W| \times|T|$ cost matrix $C$ where $C(i, j)$ is the cost of assigning element $i$ of $W$ to element $j$ of $T \stackrel{49 \mid 50}{ }$ To solve the assignment problem, the objective is to

$$
\operatorname{minimize} \sum_{i=1}^{|W|} \sum_{j=1}^{|T|} C(i, j) x_{i j}
$$

subject to

$$
\begin{aligned}
& \sum_{i=1}^{|W|} x_{i j} \leq 1, \quad(j=1, \ldots,|T|), \\
& \sum_{j=1}^{|T|} x_{i j}=1, \quad(i=1, \ldots,|W|), \\
& x_{i j} \in\{0,1\}, \quad(\forall i, j) .
\end{aligned}
$$

In Eq. (G.3), $x_{i j}$ is an indicator variable for the assignment of element $W_{i}$ to element $T_{j}$. Each element of $T$ is assigned to at most one element of $W$, i.e. each "task" cannot be assigned to more than one "worker" (Eq. [G.1)). Each element of $W$ is assigned to exactly one element of $T$ (Eq. (G.2)). The resulting linear programs can be solved with a mixed integer linear programming solver, e.g. lpSolve $\frac{52}{.}$

\section{References}

1. A. Omidvarnia, M. Pedersen, J. M. Walz, D. N. Vaughan, D. F. Abbott and G. D. Jackson, Dynamic regional phase synchrony (DRePS): An instantaneous measure of local fMRI connectivity within spatially clustered brain areas, Hum. Brain Mapp. 37(5) (2016) 1970-1985.

2. K. J. Friston, Functional and effective connectivity: A review, Brain Connect. 1(1) (2011) 13-36.

3. A. Fornito, A. Zalesky and M. Breakspear, Graph analysis of the human connectome: Promise, progress, and pitfalls, Neuroimage 80 (2013) 426444.

4. L. Leistritz, K. Schiecke, L. Astolfi and H. Witte, Time-variant modeling of brain processes, Proc. IEEE 104(2) (2016) 262-281.

5. H. E. Wang, C. G. Bénar, P. P. Quilichini, K. J. Friston, V. K. Jirsa and C. Bernard, A systematic framework for functional connectivity measures, Front. Neurosci. 8(57) (2014) 31916.

6. B. Zahneisen, T. Grotz, K. J. Lee, S. Ohlendorf, M. Reisert, M. Zaitsev and J. Hennig, Threedimensional MR-encephalography: Fast volumetric brain imaging using rosette trajectories, Magn. Reson. Med. 65(5) (2011) 1260-1268.

7. R. Hindriks, M. H. Adhikari, Y. Murayama, M. Ganzetti, D. Mantini, N. K. Logothetis and G. Deco, Can sliding-window correlations reveal dynamic functional connectivity in resting-state fMRI?, Neuroimage 127 (2016) 242-256.

8. F. De Vico Fallani, L. Astolfi, F. Cincotti, D. Mattia, M. G. Marciani, A. Tocci, S. Salinari, H. Witte, 
W. Hesse, S. Gao, A. Colosimo and F. Babiloni, Cortical network dynamics during foot movements, Neuroinformatics $\mathbf{6}(1)$ (2008) 23-34.

9. C. Koutlis and D. Kugiumtzis, Discrimination of coupling structures using causality networks from multivariate time series, Chaos 26(9) (2016) 093120 .

10. S. Fortunato and D. Hric, Community detection in networks: A user guide, Phys. Rep. 659 (2016) 1-44.

11. M. T. Schaub, J.-C. Delvenne, M. Rosvall and R. Lambiotte, The many facets of community detection in complex networks, Appl. Netw. Sci. 2(1) (2017) 4.

12. S. Fortunato, Community detection in graphs, Phys. Rep. 486(3-5) (2010) 75-174.

13. D. S. Bassett, N. F. Wymbs, M. A. Porter, P. J. Mucha, J. M. Carlson and S. T. Grafton, Dynamic reconfiguration of human brain networks during learning, Proc. Natl. Acad. Sci. USA 108(18) (2011) 7641-7646.

14. D. Vatansever, D. K. Menon, A. E. Manktelow, B. J. Sahakian and E. A. Stamatakis, Default mode dynamics for global functional integration, J. Neurosci. 35(46) (2015) 15254-15262.

15. M. Bola and V. Borchardt, Cognitive processing involves dynamic reorganization of the whole-brain network's functional community structure, J. Neurosci. 36(13) (2016) 3633-3635.

16. C. Schmidt, B. Pester, N. Schmid-Hertel, H. Witte, A. Wismüller and L. Leistritz, A multivariate Granger causality concept towards full brain functional connectivity, PLoS One 11(4) (2016) $\mathrm{e} 0153105$.

17. A. Anastasiou and E. Ifeachor, A novel thresholding method for the analysis of functional connectivity networks of the brain, in 2010 10th IEEE Int. Conf. Information Technology and Applications in Biomedicine (ITAB 2010) (IEEE, Corfu, Greece, 2010), pp. 1-6.

18. B. C. M. van Wijk, C. J. Stam and A. Daffertshofer, Comparing brain networks of different size and connectivity density using graph theory, PLoS One 5(10) (2010) e13701.

19. F. De Vico Fallani, V. Latora and M. Chavez, A topological criterion for filtering information in complex brain networks, PLoS Comput. Biol. 13(1) (2017) e1005305.

20. M. Rosvall and C. T. Bergstrom, Mapping change in large networks, PLoS One 5(1) (2010) e8694.

21. A. Mierau, B. Pester, T. Hülsdünker, K. Schiecke, H. K. Strüder and H. Witte, Cortical correlates of human balance control, Brain Topogr. 30(4) (2017) 434-446.

22. G. Gratton, M. G. H. Coles and E. Donchin, A new method for off-line removal of ocular artifact, Electroencephalogr. Clin. Neurophysiol. 55(4) (1983) 468-484.
23. L. Leistritz, B. Pester, A. Doering, K. Schiecke, F. Babiloni, L. Astolfi and H. Witte, Time-variant partial directed coherence for analysing connectivity: A methodological study, Philos. Trans. R. Soc. A 371(1997) (2013) 20110616.

24. A. Mierau, T. Hülsdünker and H. K. Strüder, Changes in cortical activity associated with adaptive behavior during repeated balance perturbation of unpredictable timing, Front. Behav. Neurosci. 9 (2015) 233.

25. T. Milde, L. Leistritz, L. Astolfi, W. H. R. Miltner, T. Weiss, F. Babiloni and H. Witte, A new Kalman filter approach for the estimation of highdimensional time-variant multivariate AR models and its application in analysis of laser-evoked brain potentials, Neuroimage 50(3) (2010) 960-969.

26. E. Ghumare, M. Schrooten, R. Vandenberghe and P. Dupont, Comparison of different Kalman filter approaches in deriving time varying connectivity from EEG data, in 2015 37th Annual Int. Conf. Engineering in Medicine and Biology Society $(E M B C)$ (IEEE, Milan, Italy, 2015), pp. 2199-2202.

27. A. Korzeniewska, M. Mańczak, M. Kamiński, K. J. Blinowska and S. Kasicki, Determination of information flow direction among brain structures by a modified directed transfer function (dDTF) method, J. Neurosci. Methods 125(1-2) (2003) 195-207.

28. F. Van de Steen, L. Faes, E. Karahan, J. Songsiri, P. A. Valdes-Sosa and D. Marinazzo, Critical comments on EEG sensor space dynamical connectivity analysis, Brain Topogr. 84 (2016) 463-412.

29. M. Kamiński and K. J. Blinowska, The influence of volume conduction on DTF estimate and the problem of its mitigation, Front. Comput. Neurosci. 11 (2017) 36.

30. T. Hülsdünker, A. Mierau, C. Neeb, H. Kleinöder and H. K. Strüder, Cortical processes associated with continuous balance control as revealed by EEG spectral power, Neurosci. Lett. 592 (2015) 1-5.

31. S. Horvath, Weighted Network Analysis (Springer, New York, Dordrecht, Heidelberg, London, 2011), DOI 10.1007/978-1-4419-8819-5.

32. Y.-R. Lin, Y. Chi, S. Zhu, H. Sundaram and B. L. Tseng, Facetnet: A framework for analyzing communities and their evolutions in dynamic networks, in Proc. 17th Int. World Wide Web Conf. (Beijing, China, 2008), pp. 685-694.

33. A. Lancichinetti and S. Fortunato, Consensus clustering in complex networks, Sci. Rep. 2 (2012) 336.

34. V. Latora and M. Marchiori, Efficient behavior of small-world networks, Phys. Rev. Lett. 87(19) (2001) 198701-198706.

35. H. Lee, H. Kang, M. K. Chung, B.-N. Kim and D. S. Lee, Persistent brain network homology from the perspective of dendrogram, IEEE Trans. Med. Imag. 31(12) (2012) 2267-2277. 
36. A. Khalid, B. S. Kim, M. K. Chung, J. C. Ye and D. Jeon, Tracing the evolution of multi-scale functional networks in a mouse model of depression using persistent brain network homology, Neuroimage 101 (2014) 351-363.

37. V. D. Blondel, J.-L. Guillaume, R. Lambiotte and E. Lefebvre, Fast unfolding of communities in large networks, J. Stat. Mech., Theory Exp. 2008(10) (2008) P10008.

38. P. Pons and M. Latapy, Computing communities in large networks using random walks, Int. Symp. Comput. Inf. Sci. 3733 (2005) 284-293.

39. M. Rosvall and C. T. Bergstrom, Maps of random walks on complex networks reveal community structure, Proc. Natl. Acad. Sci. USA 105(4) (2008) 1118-1123.

40. A. Clauset, M. E. J. Newman and C. Moore, Finding community structure in very large networks, Phys. Rev. E 70(6) (2004) 066111.

41. E. A. Leicht and M. E. J. Newman, Community structure in directed networks, Phys. Rev. Lett. 100(11) (2008) 118703.

42. M. Chen, T. Nguyen and B. K. Szymanski, On measuring the quality of a network community structure, in Int. Conf. Social Computing (SocialCom) (Alexandria, USA, 2013), pp. 122-127.

43. K. Miettinen, Nonlinear Multiobjective Optimization, International Series in Operations Research \& Management Science, Vol. 12 (Springer, 1998).

44. N. Srinivas and K. Deb, Multiobjective optimization using nondominated sorting in genetic algorithms, Evol. Comput. 2(3) (1994) 221-248.

45. P. Eskelinen and K. Miettinen, Trade-off analysis approach for interactive nonlinear multiobjective optimization, OR Spectrum 34(4) (2012) 803-816.

46. M. Ehrgott, Multicriteria Optimization, 2nd edn. (Springer, Berlin, Heidelberg, New York, 2005).

47. Y. Cao and J. Guillaume, Pareto front, https:// github.com/josephguillaume/paretoFront.

48. H. W. Kuhn, The Hungarian method for the assignment problem, Nav. Res. Logist. Q. 2(1-2) (1955) $83-97$.

49. K. C. Gilbert and R. B. Hofstra, Multidimensional assignment problems, Decis. Sci. 19(2) (1988) 306321.

50. D. W. Pentico, Assignment problems: A golden anniversary survey, Eur. J. Oper. Res. 176(2) (2007) 774-793.

51. K. Schiecke, C. Schmidt, D. Piper, P. Putsche, M. Feucht, H. Witte and L. Leistritz, Assignment of empirical mode decomposition components and its application to biomedical signals, Methods Inf. Med. 54(5) (2015) 461-473.

52. M. Berkelaar, J. Dirks, K. Eikland and P. Notebaert, lpSolve, http://lpsolve.sourceforge.net/5.5.

53. M. Meilă, Comparing clusterings - An information based distance, J. Multivariate Anal. 98(5) (2007) $873-895$.
54. P. Nachev, C. Kennard and M. Husain, Functional role of the supplementary and pre-supplementary motor areas, Nat. Rev. Neurosci. 9(11) (2008) 856869.

55. M. Rubinov and O. Sporns, Complex network measures of brain connectivity: Uses and interpretations, Neuroimage 52(3) (2010) 1059-1069.

56. M. S. Granovetter, The strength of weak ties, $A m$. J. Sociol. 78(6) (1973) 1360-1380.

57. M. S. Granovetter, The strength of weak ties: A network theory revisited, Sociol. Theory 1 (1983) 201233.

58. S. Aral, The future of weak ties, Am. J. Sociol. 121(6) (2016) 1931-1939.

59. R. S. Burt, Bridge decay, Social Netw. 24(4) (2002) 333-363.

60. D. Greene, D. Doyle and P. Cunningham, Tracking the Evolution of Communities in Dynamic Social Networks, in 2010 Int. Conf. Advances in Social Networks Analysis and Mining (ASONAM) (Odense, Denmark, 2010), pp. 176-183.

61. B. H. Good, Y.-A. de Montjoye and A. Clauset, Performance of modularity maximization in practical contexts, Phys. Rev. E 81(4) (2010) 046106.

62. J. Hopcroft, O. Khan, B. Kulis and B. Selman, Tracking evolving communities in large linked networks, Proc. Natl. Acad. Sci. USA 101(suppl 1) (2004) 5249-5253.

63. B. Karrer, E. Levina and M. E. J. Newman, Robustness of community structure in networks, Phys. Rev. E 77(4) (2008) 046119.

64. D. Gfeller, J.-C. Chappelier and P. De Los Rios, Finding instabilities in the community structure of complex networks, Phys. Rev. E 72(5) (2005) 056135 .

65. C. Schmidt, B. Pester, M. Nagarajan, H. Witte, L. Leistritz and A. Wismüller, Impact of multivariate Granger Causality analyses with embedded dimension reduction on network modules, in 2014 36th Annual Int. Conf. Engineering in Medicine and Biology Society $(E M B C)$ (IEEE, Chicago, USA, 2014), pp. 2797-2800.

66. M. Wacker and H. Witte, Adaptive phase extraction: Incorporating the Gabor transform in the matching pursuit algorithm, IEEE Trans. Biomed. Eng. 58(10) (2011) 2844-2851.

67. L. A. Baccalá and K. Sameshima, Partial directed coherence: A new concept in neural structure determination, Biol. Cybern. 84(6) (2001) 463-474.

68. S. Asur, S. Parthasarathy and D. Ucar, An eventbased framework for characterizing the evolutionary behavior of interaction graphs, ACM Trans. Knowl. Discov. Data 3(4) (2009) 16-36.

69. G. Palla, A.-L. Barabási and T. Vicsek, Quantifying social group evolution, Nature 446(7136) (2007) 664-667. 